\title{
Salmonella endorses a dormant state within human epithelial cells for persistent infection
}

Chak Hon Luk ${ }^{1,2}$, Yuen-Yan Chang ${ }^{1}$, Jost Enninga ${ }^{1,2 *}$

${ }^{1}$ Dynamics of Host-Pathogen Interactions Unit, Institut Pasteur and CNRS UMR3691, 75724 Paris, France

${ }^{2}$ Université de Paris, Sorbonne Paris Cité, Paris, France

*Corresponding author. Email: jost.enninga@pasteur.fr (J.E.)

Short title: Salmonella dormancy in enterocyte 


\begin{abstract}
Salmonella Typhimurium (S. Typhimurium) is an enteric bacterium capable of invading a wide range of hosts, including rodents and humans. It targets different host cell types showing different intracellular lifestyles. Within the infected cells $S$. Typhimurium colonizes multiple intracellular niches, and it is able to either actively divide at various rates, or remain dormant to persist. A comprehensive tool to monitor these distinct $S$. Typhimurium lifestyles has not been available so far. Here we developed a novel fluorescent reporter, Salmonella Intracellular Analyzer (SINA), compatible for fluorescence microscopy and flow cytometry for quantification at the single-bacterium level. Using SINA, we identified a $S$. Typhimurium subpopulation in infected epithelial cells that exhibits a unique phenotype in comparison to the previously documented vacuolar or cytosolic $S$. Typhimurium. This newly identified subpopulation remained dormant within a vesicular compartment distinct from either conventional Salmonella-containing vacuoles (SCV) or the previously reported niche of dormant $S$. Typhimurium inside macrophages. The dormant $S$. Typhimurium inside enterocytes were viable and expressed Salmonella Pathogenicity Island 2 (SPI-2) virulence factors at later infection time points. We found that the formation of these dormant $S$. Typhimurium is not triggered by the loss of SPI-2 expression but it is regulated by (p)ppGpp-mediated stringent response through RelA and SpoT. We predict that intraepithelial dormant $S$. Typhimurium represents an important pathogen niche as it provides an alternative strategy for $S$. Typhimurium pathogenicity and persistence.
\end{abstract}

\title{
Author Summary
}

Salmonella Typhimurium is a clinically relevant bacterial pathogen that causes Salmonellosis. It can actively or passively invade various host cell types and reside in a Salmonella-containing vacuole (SCV) within host cells. The SCV can be remodeled into a replicative niche with the aid of Salmonella Type III Secretion System 2 (T3SS2) effectors or else, the SCV is ruptured for the access of the nutrient-rich host cytosol. Depending on the infected host cell type, $S$. Typhimurium undertake different lifestyles that are distinct by their subcellular localization, replication rate and metabolic rate. We present here a novel fluorescent reporter system that rapidly 
detects $S$. Typhimurium lifestyles using fluorescence microscopy and flow cytometry. We identified a dormant $S$. Typhimurium population within enterocytes that displays capacities in host cell persistence, dormancy exit and antibiotic tolerance. We found that the molecular pathway suppressing $S$. Typhimurium dormancy in enterocytes is the one that has been shown to promote dormancy in macrophages. This suggests a divergent physiological consequence regulated by the same set of $S$. Typhimurium molecular mediators depending on the challenged host cell type. Altogether, our work demonstrates the potential of fluorescence reporters in facile bacterial characterization, and revealed a dormant $S$. Typhimurium population in human enterocytes that is distinct from those observed in macrophages and fibroblasts. 


\section{Introduction}

Salmonella enterica serovar Typhimurium (S. Typhimurium) is an enteric bacterium that closely associates with global food-borne illnesses. The prevalence of $S$. Typhimurium has placed a severe burden on healthcare and the global food industry $[1,2]$. S. Typhimurium resides in different natural reservoirs and is transmitted to humans through contaminated food.

Upon arrival in the human intestine after ingestion, a portion of the luminal $S$. Typhimurium expresses the Type III Secretion System 1 (T3SS1) and its cognate effectors encoded in Salmonella Pathogenicity Island 1 (SPI-1) to induce its active entry into non-phagocytic epithelial cells. $S$. Typhimurium also targets other cell types, such as fibroblasts and macrophages. During these events, it induces local tissue injuries and eventually breaches the intestinal barrier to reach the lamina propria and tissue-resident immune cells. Then, $S$. Typhimurium is carried by macrophages to mesenteric lymph nodes and eventually to the liver and spleen for persistent infection (-reviewed in Ilyas et al. 2017).

Within enterocytes $S$. Typhimurium are encapsulated in an endocytic compartment coined Salmonella-containing vacuole (SCV) that matures by acidification within the first hours of internalization. The reducing $\mathrm{pH}$ and changing osmolarity of SCV induce the shutdown of T3SS1 and expression of a second T3SS, T3SS2, from Salmonella Pathogenicity Island 2 (SPI-2). The T3SS2 effectors remodel the SCV into a viable niche for $S$. Typhimurium replication [4,5]. Default maturation of the $\mathrm{SCV}$ is marked by the sequential acquisition and removal of endocytic trafficking markers, such as the small GTPase RAB5 and RAB7 as well as Lysosome-associated membrane glycoprotein 1 (LAMP1) [6]. During these events, the SCV dynamically interacts with the surrounding macropinosomes, which controls SCV stability. Consequently, $S$. Typhimurium can reside either in a remodeled SCV or disrupt the SCV exhibiting distinct replication rates and specific metabolic activity [7-9].

Differential lifestyles are also known for $S$. Typhimurium infecting other cell types. In fibroblasts, the SCV associates with the aggrephagy machinery that either clears the infection or allows $S$. Typhimurium to putatively persist in the cell [10]. In macrophages, $S$. Typhimurium expresses the T3SS2 to remodel the SCV immediately 
after bacterial entry, or the pathogen adopts a dormant behavior mediated by toxinantitoxin (TA) system [11,12].

Persistence and relapse of $S$. Typhimurium infection due to the failure of bacterial eradication with antibiotics has been tied to $S$. Typhimurium dormancy. Numerous antibiotics target major active machineries, including DNA replication, transcription and translation of extracellular bacteria, therefore dormant intracellular pathogens appear to be less or not susceptible to such treatments [13]. S. Typhimurium dormancy and antibiotics persistence have been reported in macrophages regulated by the Guanosine pentaphosphate (ppGpp) stringent response pathway. The two (p)ppGpp synthases RelA and SpoT control the bacterial (p)ppGpp level, which regulates in turn the activity of the ATP-dependent protease Lon to degrade the antitoxin and release the toxin TacT for arresting protein translation. The arrest of translation by TacT leads to a halt of bacterial growth giving rise to the insensitivity and tolerance towards antibiotics [12,14]. Despite reports of $S$. Typhimurium antibiotics persistence in the epithelium and lamina propria of the mouse intestine, it is not clear whether this involves dormant bacteria [15].

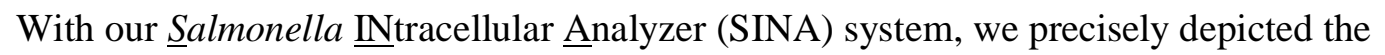
intracellular bacterial lifestyles at the single bacterium level, identifying a novel $S$. Typhimurium population within enterocytes that is dormant. Dormant persisters within enterocytes are localized in a unique vacuolar compartment different from the one described in macrophages. We found that T3SS2 expression and the Lon protease are dispensable for this new $S$. Typhimurium population, while the bifunctional enzyme SpoT and monofunctional enzyme RelA negatively regulate $S$. Typhimurium dormancy in epithelial cells. 


\section{Results}

Development of a multiplex fluorescent reporter series, the Salmonella

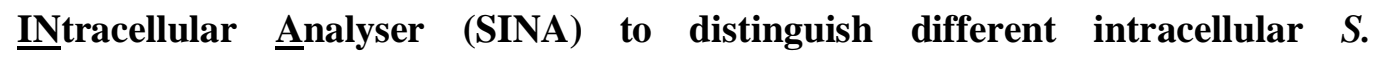
Typhimurium lifestyles

The distinct intracellular lifestyles of $S$. Typhimurium upon invasion of epithelial cells have been described either with regard to the specific pathogen localization or with regard to the bacterial growth dynamics. To date, fluorescent reporters are available to identify $S$. Typhimurium within vacuolar and cytosolic localizations; while the others measure the replication rate of the pathogen [16-18]. However, these localization and replication-rate reporters have not been coupled, as it has been generally assumed that the bacterial localization determines its replication rate. This notion has been challenged by different reports, for example on the different growth rates of $S$. Typhimurium within the cytosol depending on the targeting by autophagy [19-23]. A combined reporter system would enable a comprehensive elucidation of the intracellular lifestyle of a given intracellular pathogen. Therefore, we developed a

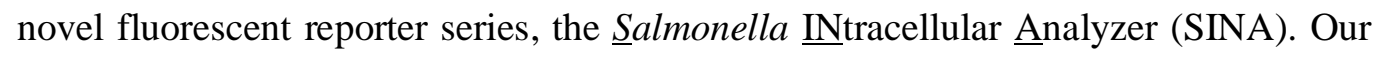
SINA1.1 reporter is composed of two separated modules to indicate the bacterial localization and replication rate. The localization module consists of two transcription reporters driven by localization-specific promoters, while the replication rate module carries a constitutively expressed fluorescent timer (Fig 1A, S1 Fig). At the molecular level, the localization module is composed of vacuolar (Vac) and cytosolic (Cyt) submodules, which utilize two characterized promoters, $\mathrm{P}_{\text {ssaG }}$ and $\mathrm{P}_{u h p T}$ to drive the expression of tagBFP and smURFP, respectively [16,17]. We confirmed the functionality of the fluorescent $\mathrm{P}_{s s a G}$ and $\mathrm{P}_{u h p T}$ reporters for our experimental setup during $S$. Typhimurium invasion of epithelial cells using a digitonin assay measured by flow cytometry (S2-3 Figs). The replication rate module encodes $\operatorname{Timer}^{\mathrm{bac}}$, a DsRed mutant (S197T), which has been previously employed to differentiate $S$. Typhimurium subpopulations by their replication rates [18]. The emission spectrum of Timer ${ }^{\text {bac }}$ shifts from green to red as it matures, which reflects the bacterial metabolic activity (change in slope, Fig 1B top) as well as the replication rate (unvarying slope, varying green:red ratio, Figure 1B bottom) [24]. When Timer ${ }^{\text {bac }}$ is constitutively expressed, a metabolically active $S$. Typhimurium bacterium emits both green and red signals resulting from immature green and maturing red fluorophores. In case such a bacterium experiences a metabolic halt, it eventually emits only red 
signals, due to the maturation of the existing green fluorophores in concert with ceased de novo synthesis of the green fluorophores. With SINA, we are able to simultaneously collect information on these replication rate changes of $S$. Typhimurium and its localization at single bacterium resolution, which enables a comprehensive and quantitative reflection of $S$. Typhimurium physiology inside an infected host.

To validate the functionality of our SINA system during $S$. Typhimurium invasion of epithelial cells, we employed fluorescence microscopy and FACS analysis (Fig 1C). With fluorescence microscopy, we observed intracellular $S$. Typhimurium simultaneously emitting both green and red signals (Timer $\left.{ }^{\mathrm{bac}}\right)$, but not $\mathrm{Vac}\left(\mathrm{P}_{s s a G^{-}}\right.$

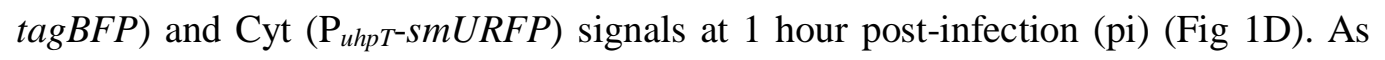
these bacteria committed to vacuolar or cytosolic lifestyles at 6 hours pi, we observed that $S$. Typhimurium in cells with $<10$ bacteria emitted Vac signal during this time course. On the other hand, we observed a mixed population of $S$. Typhimurium in cells with $>10$ bacteria, where clusters of $\mathrm{Cyt}^{+} S$. Typhimurium of low $\mathrm{Timer}^{\text {bac }}$ signals (arrow) and individual $\mathrm{Vac}^{+} S$. Typhimurium (arrowhead) were detected. In the cells containing mixed $S$. Typhimurium populations, bacteria were either $\mathrm{Vac}^{+}$or $\mathrm{Cyt}^{+}$but not double positive, showing the presence of two populations with distinct discernible lifestyles (Fig 1D). We were also able to track the onset of bacterial division and signal output from SINA by time-lapse microscopy (S1-3 Movies).

We devised a gating strategy to quantitatively analyze the SINA reporter output as readout for the bacterial lifestyles in single $S$. Typhimurium-infected cells using FACS (S2 Fig). In brief, we first defined the infected cells by the size of the analyzed events (under SSC-A vs FSC-A plot), followed by the positive signals in $\operatorname{Timer}^{580}$ vs Timer ${ }^{510}$ plot (i.e. cells harboring $S$. Typhimurium). We then further classified the $S$. Typhimurium-infected cells into four sub-types according the signals of the localization module (tagBFP::SPI-2 vs smURFP::cytosolic plot), corresponding to cells with either vacuolar bacteria $\left(\mathrm{Vac}^{+} \mathrm{Cyt}\right)$ or cytosolic bacteria $\left(\mathrm{Vac}^{-} \mathrm{Cyt}^{+}\right)$or cells with both vacuolar and cytosolic populations $\left(\mathrm{Vac}^{+} \mathrm{Cyt}^{+}\right)$or cells harboring $S$. Typhimurium that express only basal levels of the Vac and Cyt signals (S2 Fig). We observed that intracellular $S$. Typhimurium behaved as a population with a homogenous replication rate and basal expression levels of Vac and Cyt at 1 hour pi 
(Fig 1E). At 6 hours pi, this homogenous population segregated into $\mathrm{Vac}^{+}$and $\mathrm{Cyt}^{+}$ subpopulations, with a $\mathrm{Cyt}^{+}$distribution similar to that reported in literature $(10-20 \%$ cytosolic) (Fig 1E) [19]. The gradual separation of these subpopulations could be detected with SINA1.1 throughout the course of infection (S4 Fig). As we backgated the infected cells, we observed either cells harboring only vacuolar $S$. Typhimurium $\left(\operatorname{Vac}^{+} \mathrm{Cyt}\right)$, and some cells with both vacuolar and cytosolic bacteria $\left(\mathrm{Vac}^{+} \mathrm{Cyt}^{+}\right)$, each of them formed a distinct population of different Green:Red ratio on the plot of Timer $^{510}$ against Timer $^{580}$ (S5A Fig). Together, this demonstrated that our novel SINA1.1 reporter is capable of simultaneously and quantitatively distinguishing the $S$. Typhimurium lifestyles by their subcellular localization and replication rate at both single infected cell and single bacterium level using flow cytometry and fluorescence microscopy. The combination of the SINA reporter with FACS fosters higher throughput analysis of $S$. Typhimurium lifestyles in infected cells as compared to microscopy, extending the possibility for rapid screening.

\section{A novel dormant $S$. Typhimurium subpopulation in human epithelial cell}

With the SINA1.1 reporter, we used the SPI-2 expression module to distinguish vacuolar $S$. Typhimurium from cytosolic bacteria. In the plot of the localization module, we identified an easily discernable population ( $5-10 \%)$ of infected epithelial cells harboring $\mathrm{Vac}^{-} \mathrm{Cyt}^{-} S$. Typhimurium detectable as early as 2 hours pi, which became apparent at 6 hours pi (Fig 2A, S6 Fig). We backgated the $\mathrm{Vac}^{-} \mathrm{Cyt}^{-}$ population, and we extracted physical parameters from the Timer ${ }^{\text {bac }}$ plot. This revealed that the $\mathrm{Vac}^{-} \mathrm{Cyt}^{-} S$. Typhimurium exhibit a similar replication rate (S5A Fig) but a reduced metabolic activity (S5B Fig) compared to $\mathrm{Vac}^{+} \mathrm{Cyt}{ }^{-} S$. Typhimurium as depicted by the green:red ratio and slope of Timer ${ }^{\text {bac }}$ plot, respectively. The capacities of Timer ${ }^{\text {bac }}$ in the measurement of bacterial replication rate and metabolism have been well-elaborated in previous applications [18,25]. This $\mathrm{Vac}^{-} \mathrm{Cyt}^{-} S$. Typhimurium population was also visualized using live microscopy to confirm their presence using different detection approaches (S3 Movie). This was intriguing as metabolically inactive $S$. Typhimurium have not been reported in enterocytes so far. We thus infected polarized intestinal epithelial Caco-2 monolayers, and confirmed the presence of the $\mathrm{Vac}^{-} \mathrm{Cyt}^{-}$subpopulation of shifted metabolic profile in a cellular model system for intestinal infections (S7 Fig). We also performed control infections in differentiated THP-1 cells to test the sensitivity of SINA1.1 in observing dormant 
S. Typhimurium in this macrophage model as described before (S8 Fig). To determine the intracellular localization of $\mathrm{Vac}^{-} \mathrm{Cyt}^{-} S$. Typhimurium, we further performed a digitonin assay localizing this subpopulation to a host vesicular compartment (S3A-F Fig) [19]. Together, these results demonstrated the presence of a novel intracellular $S$. Typhimurium population within epithelial cells that exhibits a lowered metabolic rate and resides in a host vesicular compartment, implicating a putative dormant phenotypic variant.

Intracellular $S$. Typhimurium encounters a number of stresses upon uptake into host cells, including oxidative, $\mathrm{pH}$ and osmotic stress, which serve as key signals to trigger transcription reprogramming for the adaptation of an intra-host environment [26]. During infection of macrophages, the SCV microenvironment drives a portion of $S$. Typhimurium into a dormant state that contributes to the elevation of antimicrobial persistence and polarization of infected macrophage [12,27]. To characterize whether Vac $^{-} \mathrm{Cyt}^{-} S$. Typhimurium shares similar physiologies with dormant $S$. Typhimurium inside macrophages, we set out to determine the metabolic state of the $\mathrm{Vac}^{-} \mathrm{Cyt}^{-}$ population. By replacing the cytosolic submodule of SINA1.1 with an arabinose inducible cassette to generate SINA1.5, we measured S. Typhimurium's capacity to respond to arabinose treatment. This modification enabled us to directly monitor the metabolic activity of $S$. Typhimurium during the infection process (Fig 2B). The response of intracellular bacteria towards extracellular arabinose induction has been reported previously to characterize the metabolic state of macrophage-borne dormant $S$. Typhimurium [11]. With SINA1.5, we observed that approximately half of the $\mathrm{Vac}^{-}$ $S$. Typhimurium did not respond to arabinose induction at designated time intervals (Fig 2C). Combining this with our observations on the reduced metabolism (S5B Fig), we were able to put forward the first evidence to propose that the $\mathrm{Vac}^{-} \mathrm{Cyt}^{-} S$. Typhimurium adopts a dormant state (coined as dormant $S$. Typhimurium hereafter) upon their internalization into epithelial cells. We also addressed whether this dormant phenotype confers a reduced sensitivity towards antibiotics. To test this, we supplemented ciprofloxacin (CIP) to the infected cell and determined the viability of dormant $S$. Typhimurium by CFU. We observed a higher survival rate of dormant $S$. Typhimurium as compared to vacuolar $S$. Typhimurium, similar to that observed in the murine intestine (Fig 2D) [15]. Thus, the newly identified dormant $S$. Typhimurium population within enterocytes is less susceptible to antibiotic treatment. 


\section{Dormant $S$. Typhimurium resides in a unique vesicular compartment}

We set out to determine whether the dormant $S$. Typhimurium localization is distinct from conventional SCVs. To determine this by immunofluorescence staining, we simplified SINA1.1 to SINA1.4 to free the red and far-red channels for indirect immunofluorescence staining of selected endocytic markers (Fig 2B). LAMP1 labels host lysosomes as well as the matured SCV, which is also present on the SCV of dormant $S$. Typhimurium in macrophages [6,11]. By fluorescence microscopy, we only observed minor recruitment of LAMP1 to the proximity of dormant $S$. Typhimurium within epithelial cells, in contrast to the high LAMP1 incidence proximal to $\mathrm{Vac}^{+} S$. Typhimurium (Fig 3A and B). To address if this dormant population is targeted by host autophagy, we next analyzed the localization of the autophagy marker LC3. Intriguingly, we did not detect any localization of LC3 proximal to the majority of the dormant $S$. Typhimurium (Fig 3A and B). Therefore, we conclude that dormant $S$. Typhimurium are localized within a unique membranebound compartment distinct from the conventional SCV and that of dormant $S$. Typhimurium in macrophages, suggesting such dormancy formation is heavily governed by endocytic trafficking $[11,28]$.

\section{Dormant $S$. Typhimurium are viable, cultivable, resume metabolism and express virulence genes in host cells.}

Endocytic vesicles are either recycled or undergo fusion with the lysosomes for degradation. The same fate also applies to vacuolar $S$. Typhimurium, where SPI-2 deficient strains have a reduced survival capacity compared to SPI-2 competent strains [29]. We collected the infected cells harboring dormant $S$. Typhimurium by cell sorting at $>90 \%$ purity and plated them for colony forming unit (CFU) measurement, a classical approach to determine the viability of the intracellular $S$. Typhimurium. We observed that dormant $S$. Typhimurium are viable and cultivable (Fig 4A), contrasting to the viable but not cultivable nature of dormant $S$. Typhimurium in murine macrophages $[11,12]$. To determine the fate of the dormant $S$. Typhimurium, we enriched and plated the viable cells harboring dormant $S$. Typhimurium, and monitored the bacterial behavior at 24 hours pi (S9 Fig). We observed that $50 \%$ of the dormant $S$. Typhimurium in infected cells collected at 6 hours pi became metabolically active and expressed SPI-2 at 24 hours pi, as 
demonstrated by the population shift in the Timer ${ }^{\text {bac }}$ plot, and becoming $\mathrm{Vac}^{+}$(Fig 4B). To determine if the dormant $S$. Typhimurium persists in the host, we further enriched infected cells harboring dormant $S$. Typhimurium and monitored the presence and the viability of dormant $S$. Typhimurium at 7 days pi. The dormant $S$. Typhimurium were found to persist in cells and remained viable and cultivable over the whole period of 7 days (Fig 4A, “168h”).

These results demonstrated that dormant $S$. Typhimurium are viable, they exhibit a delayed expression of SPI-2, and they persist in the epithelial host cells for up to 7 days. Such unique metabolic and virulence reprogramming could serve as a strategic step for intestine-borne $S$. Typhimurium to prolong gut inflammation for community benefits and reservoir for relapse [30].

\section{S. Typhimurium dormancy does not rely on the loss of SPI-2 expression, but is regulated by (p)ppGpp biogenesis.}

We then studied whether the lack of SPI-2 expression drives S. Typhimurium dormancy in epithelial cells. Using our SINA1.1 reporter in the S. Typhimurium SPI2 secretion deficient mutant $\Delta s s a V$ [31], we observed no significant difference in the proportion of $\mathrm{Vac}^{-} \mathrm{Cyt}^{-} S$. Typhimurium between wild type and the $\Delta s s a V$ mutant (Fig $5 \mathrm{~A})$. This indicates that the formation of dormant $S$. Typhimurium is not a consequence caused by the lack of SPI-2 expression or T3SS2 effectors secretion during the infection of epithelial cells.

Class II toxin-antitoxin (TA) systems regulate the formation of non-pathogenic E. coli in laboratory conditions. TA systems are comprised of a toxin and an antitoxin that counter-balances the toxin to regulate bacterial physiology, including growth arrest. A major TA system involves the stringent response mediated by the monofunctional (p)ppGpp synthases RelA and bifunctional (p)ppGpp synthases SpoT, after which (p)ppGpp binds to DksA to mediate transcription reprogramming for bacterial adaptation. The surge in (p)ppGpp levels also activates the ATP-dependent Lon protease to degrade Type II antitoxins to release the free toxins [32-34]. In recent reports, stringent response has been associated with slow growing $S$. Typhimurium populations, and TA systems are implicated in the $S$. Typhimurium persistence in macrophages $[12,14]$. Therefore, we assessed the links between the stringent response 
and $S$. Typhimurium dormancy in epithelial cells, studying the mutant strains (i) $\triangle$ relA ((p)ppGpp synthase), (ii) $\triangle r e l A \triangle s p o T$ ((p)ppGpp synthases), (iii) $\triangle d k s A$ ((p)ppGpp-binding transcription regulator) and (iv) Slon (protease targeting antitoxin). With the $\Delta$ lon mutant, we did not observe any difference in the level of dormant $S$. Typhimurium population in infected cells ( 5-10\%), suggesting that Lon protease is dispensable for $S$. Typhimurium dormancy in epithelial cells (Fig 5C). As $\triangle$ relAspoT and $\triangle d k s A$ were reported to suffer reduced invasiveness in epithelial cells due to the reduced SPI-1 expression, we decided to construct SINA1.9 (Fig 5B), a derivative of SINA1.1 with an additional cassette for an inducible expression of hilA to compensate the reduced invasiveness of the mutants (S10 Fig) [35]. With the SINA1.9-complemented mutant strains, we obtained rescued cell invasiveness as compared to wild type $S$. Typhimurium. This allowed us to address the requirement of (p)ppGpp biogenesis and (p)ppGpp-regulated transcription for $S$. Typhimurium persistence. A significant increase in the $\mathrm{Vac}^{-} \mathrm{Cyt}^{-}$population was observed in ArelAspoT, whereas the increment was less pronounced in the $\Delta$ relA single mutant and was indifferent in $\triangle d k s A$ mutant, when comparing with the wild type strain (Fig 5C and 5D, Left and Middle panel). We further confirmed that the $\mathrm{Vac}^{-} \mathrm{Cyt}^{-}$ population of $\triangle$ relAspoT shared a comparable metabolic profile as the one observed in the wild type strain (Figure 5D, Right panel).

Together, these results suggested that (p)ppGpp stringent response mediated by SpoT but not RelA is required to restrict dormancy entry of $S$. Typhimurium within epithelial cells independent of the DksA regulon, while SPI-2 effector expression and secretion and Lon protease are dispensable.

\section{Discussion}

$S$. Typhimurium has been reported to survive in different host cells by adopting distinctive metabolic profiles, subcellular localizations and replication rates, which has also been proposed to account for various clinical complications. Herein, we report a dormant population of $S$. Typhimurium residing in a unique vesicular compartment in epithelial cells of the intestine. These dormant epithelial $S$. Typhimurium persist within host cells for a prolonged period. The SINA reporter system was instrumental for the discovery of enterocyte-borne dormant $S$. 
Typhimurium as it allowed the simultaneous depiction of the metabolism, subcellular localization and replication rate of the intracellular bacteria. The compatibility of the SINA system with microscopy and flow cytometry offers the opportunity for multiomics analysis as well as high-throughput genetic and chemical screenings on the influence of bacterial pathophysiology.

The dormant $S$. Typhimurium within epithelial cells, while remaining viable in the absence of SPI-2 expression, reside in a unique vesicular compartment distinct from the LAMP1-labelled SCV or the LC3-positive autophagosomes. Upon endocytosis, endosomes are either recycled or matured and eventually degraded via fusion with lysosomes. The SCV shares such a fate if T3SS2 effectors are not secreted to hijack the vesicular maturation pathway [31]. Therefore, we propose that dormant $S$. Typhimurium reside in a vesicular compartment idle to endocytic trafficking pathways that are independent of SPI-2 expression. The precise nature of this compartments remains to be investigated in detail. Subsequent resumption of metabolism and SPI-2 expression potentially serve as a signal to reengage the dormant membrane-enclosed $S$. Typhimurium with endocytic trafficking pathways for remodeling this novel dormant bacteria containing SCV into the default replicative niche (Fig 6A). The persistence of dormant $S$. Typhimurium in host cell for up to at least 7 days in our tested condition is also striking as the bacteria inside this vacuolar compartment possible have restricted access to the extracellular nutrients.

$S$. Typhimurium has been reported previously to enter dormant or persistent states within a modified SCV from a range of host cell types, including macrophages and fibroblasts $[10,11]$. Considering the presumably identical $S$. Typhimurium dormancy observed across the different cell models, there are substantial distinctions among the targeted host cell types in terms of the detection approaches and bacterial physiology. The first $S$. Typhimurium persisters were identified in macrophages using a dilution reporter on non-replicating $S$. Typhimurium, which enters dormancy and a viable-butnot-cultivable state upon entry [11]. The dormancy is regulated by the TA system toxin, TacT that halts protein translation and induces antibiotic persistence, where the $S$. Typhimurium subsequently exits dormancy and activates SPI-2 [14,27]. The $S$. Typhimurium persisters in fibroblast were later reported, where $S$. Typhimurium activates SPI-2 to remodel the SCV and interacts with endocytic trafficking pathways. 
The SCV subsequently interacts with host aggrephagy, where majority of the SCV S. Typhimurium are eradicated whereas the remaining $S$. Typhimurium were proposed to persist in the host. However, limited evidence regarding the viability, persistence, pathological implication as well as the underlying mechanism of persistence have been presented to date [10]. In epithelial cells, the reported $S$. Typhimurium dormancy by us is distinct from that in fibroblast and macrophage. This discrepancy lies in the commence of dormancy, capacity to replicate and permeability to the surrounding microenvironment [11]. The distinct niches of dormant $S$. Typhimurium may reflect cell-type specific vesicular trafficking, for example SCV maturation in these different target cells is not identical. Alternatively, it is possible that the way of entry impacts the development of dormant $S$. Typhimurium. Epithelial dormant $S$. Typhimurium is independent of Lon protease and is negatively regulated by SpoT, contrasting to that in macrophage that requires Lon protease, (p)ppGpp synthases RelA and SpoT $[12,34]$. The substantial difference between the $S$. Typhimurium dormancy sheds light on their potentially diverge pathophysiological implications as well as the molecular cue and mechanism that signal the establishment and exit of dormancy. It will be interesting to investigate why these regulatory modules are differentially involved in the formation of dormant $S$. Typhimurium in the different cell types, and whether the distinct niche impacts their expression and implication.

(p)ppGpp, is a bacterial alarmone that functions as a key regulator of bacterial physiology. The (p)ppGpp-mediated stringent response has been closely associated with antibiotics persistence via inhibition of protein synthesis and transcription reprogramming [36-38]. The persistent $S$. Typhimurium in macrophages is dependent on a (p)ppGpp-Lon protease-Class II TA systems axis, where TacT leads to a halt in protein translation [14]. In non-pathogenic E. coli and S. Typhimurium models, the loss of Lon and its downstream regulated TA systems leads to a diminished persister formation due to the inactivity of toxins [39]. In our findings, the dormant phenotype is negatively regulated by (p)ppGpp synthases SpoT and partially by RelA, but is independent of DksA and Lon protease-mediated pathways (Fig 6A). Our finding contrasts the current understanding on the role of stringent response on bacterial persistence, where stringent response is activated by various stress signals and (p)ppGpp synthesis would act on its molecular target to achieve persistence. Therefore, we suggest that bifunctional SpoT is required while monofunctional RelA 
is dispensable in $S$. Typhimurium dormancy in enterocytes, which echoes the previous report on the requirement of SpoT but not RelA in $S$. Typhimurium invasion and colonization of an in vivo model [35]. The essence of SpoT but not RelA for $S$. Typhimurium dormancy could suggest that either or both the (p)ppGpp hydrolysis and synthase function is required, or relA is not expressed during the course of infection. Considering that $S$. Typhimurium dormancy is independent of DksA and Lon, it implies that dormancy is likely to be mediated by pathways independent of DksA transcription reprogramming and Lon protease-mediated degradation. As RelA and SpoT function to convert GDP and GTP to (p)ppGpp, and SpoT hydrolyzes (p)ppGpp to give GTP/GDP and pyrophosphate, an imbalance of RelA/SpoT activity upsets the bacterial energy status, which could potentially act as a cue for dormancy.

With the traits we uncovered in the dormant $S$. Typhimurium within epithelial cells, this population could represent the intestinal persister, given the close association between bacterial dormancy and antibiotic persistence. Besides the proposed antibiotic persistence and horizontal gene transfer, the physiological features of enterocyte-borne dormant $S$. Typhimurium could also provide two plausible benefits to $S$. Typhimurium colonization of the host gut [40] (Fig 6B): 1) Dormancy and delayed expression of SPI-2 allow S. Typhimurium to evade cellular immunity during early invasion and to provide a sustained and extended SPI-2 expression at tissue scale, where $S$. Typhimurium reactivated from dormancy supports SPI-2 expression as classic vacuolar $S$. Typhimurium is eradicated. The sustained SPI-2 expression fuels gut inflammation to release electron acceptors for $S$. Typhimurium survival benefits in the gut lumen [30]. 2) Persistent $S$. Typhimurium within the intestinal tissue could serve as the source of subsequent infection relapse or systemic spread, where the maximum duration of persistence and molecular cues for reactivation remain to be elucidated (Fig 6B).

The cell type-specific role of the alarmone pathway on dormancy regulation indicates that the micro-environment in different cell types serves as the unique molecular cues for $S$. Typhimurium lifestyles. The generation of phenotypic variants in different cell types and host tissues potentially represent a sophisticated strategy of pathogens to evade the host defense and antibiotics treatment as well as offering community-level 
bioRxiv preprint doi: https://doi.org/10.1101/2020.11.14.382689; this version posted November 15,2020 . The copyright holder for this preprint (which was not certified by peer review) is the author/funder, who has granted bioRxiv a license to display the preprint in perpetuity. It is made available under aCC-BY-NC-ND 4.0 International license.

benefits [15]. Overall, our work highlights the importance of phenotypic heterogeneity in pathogens and its link with the pathophysiological outcomes. 


\section{Materials and methods}

\section{Mammalian cell culture}

HeLa cervical adenocarcinoma cells, Caco-2 colorectal adenocarcinoma cells and THP-1 acute monocytic leukemia cells were purchased from American Type Culture Collection (ATCC) and used within 20 passages of receipt. HeLa cells were cultured in Dulbecco's Modified Eagle Medium (DMEM, high glucose, GlutaMAX ${ }^{\mathrm{TM}}$ Supplement, ThermoFisher) containing $10 \%$ (v/v) heat-inactivated fetal bovine serum (FBS, Sigma) and incubated at $37{ }^{\circ} \mathrm{C}$ with $5 \% \mathrm{CO}_{2}$ and $100 \%$ humidity. Caco-2 cells were cultured in DMEM containing 10\% FBS, 1\% Non-essential amino acids (Gibco), 1\% HEPES (Gibco), 1\% Penicillin/Streptomycin (Gibco) and incubated at $37{ }^{\circ} \mathrm{C}$ with $5 \% \mathrm{CO}_{2}$ and $100 \%$ humidity. THP-1 cells were cultured in RPMI-1640 medium (ThermoFisher) containing $10 \% \mathrm{FBS}$ and incubated at $37{ }^{\circ} \mathrm{C}$ with $5 \% \mathrm{CO}_{2}$ and $100 \%$ humidity. HeLa and Caco-2 cells were seeded in 12-well tissue-culture treated plates (Corning Costarß) at a density of $9 \times 10^{4}$ cells/well 48 hours prior to infection. THP-1 cells were seeded in 12-well tissue-culture treated plates at a density of $9 \times 10^{4}$ cells/well 96 hours prior to infection, and differentiated in $50 \mu \mathrm{g} / \mathrm{mL}$ phorbol 12-myristate 13-acetate (PMA, Sigma) for 24 hours, and incubated in RPMI-1640 + $10 \%$ for 72 hours. For immunofluorescence staining, HeLa cells were seeded on UVtreated glass coverslips (Marienfeld) in 12-well plates 48 hours prior to infection. For cell sorting experiments, HeLa cells were seeded in $10 \mathrm{~cm}$ tissue-culture treated dishes (Corning Costar®) at a density of $1.8 \times 10^{6}$ cells/well 48 hours prior to infection.

\section{Bacterial strains}

Bacterial strains and plasmids used in this study are listed in Supplementary Table 1 and 2, respectively. All mutants were constructed using bacteriophage $\lambda$ red recombinase system from parental stain $S$. Typhimurium Typhimurium strain SL1344 using primers listed in Supplementary Table 3 [41]. Bacteria were cultured in Lysogeny broth (LB) supplemented with appropriate antibiotics, where necessary (Ampicillin $100 \mu \mathrm{g} / \mathrm{mL}$; Kanamycin $50 \mu \mathrm{g} / \mathrm{mL}$ ).

\section{Plasmid construction}

The replication rate module, Timer ${ }^{\text {bac }}$ is a generous gift from Dr. Dirk Bumann (University of Basel, Switzerland) [18]. To construct the localization module, tagBFP 
was amplified from pHRdSV40-NLS-dCas9-24xGCN4_v4-NLS-P2A-BFP-dWPRE using primers tagBFP_fw and tagBFP_rv, and replaced the GFP in pM973 to yield vacuolar module $\left(\mathrm{pP}_{s s a G^{-}} \operatorname{tag} B F P\right)$ [17,42]. For the cytosolic module $\left(\mathrm{pP}_{u h p T^{-}}\right.$ smURFP), smURFP-HO-1 and uhpT promoters were amplified from pBAD smURFP-HO-1 (smURFP_fw and smURFP_rv) and $S$. Typhimurium gDNA (uhpT_fw and uhpT_rv), respectively, and replaced the $s f G F P$ and $m x i E$ promoter in pTSAR1 [43,44]. The vacuolar (Vac_fw and tagBFP_rv) and cytosolic (uhpT_fw and Cyt_rv) modules were amplified and inserted into EcoRV and SmaI sites, respectively, of pBlueScript II KS (+) to generate pSINA-int. The localization module on pSINA-int was excised and inserted between SalI and SphI sites of pBR322 Timer $^{\text {bac }}$ to yield pSINA1.1. pSINA1.4 was generated by replacing Timer ${ }^{b a c}$ with GFP in pBR322 Timer $^{\text {bac }}$ and inserted the vacuolar module at the SalI and SphI sites. pSINA1.5 was generated by inserting the amplified inducible smURFP cassette (Ara_fw and Ara_rv) and vacuolar cassette between the SalI and EagI sites of pBR322 Timer $^{\text {bac }}$. pSINA1.7 was constructed by reverting Timer ${ }^{b a c}$ to DsRed by sitedirected mutagenesis using DsRed_fw and DsRed_rv. pBAD hilA was generated by inserting the amplified hilA (hilA_fw1 and hilA_rv1) between the BamHI and PmeI sites of pBAD smURFP-HO-1. The inducible hilA cassette was amplified using primer hilA_fw2 and hilA_rv2 and inserted into the EcoRV site of pSINA1.1 to generate pSINA1.9.

\section{Bacterial infections}

Bacteria strains were streaked from glycerol stock on LB agar plates with appropriate antibiotics 2 days prior to infection. Three bacterial colonies were picked for overnight culture in $\mathrm{LB}$ medium supplemented with $0.3 \mathrm{M} \mathrm{NaCl}$ with shaking at $37^{\circ} \mathrm{C}$. $150 \mu \mathrm{L}$ overnight culture was subculture in $3 \mathrm{~mL} \mathrm{LB}+0.3 \mathrm{M} \mathrm{NaCl}(1: 20$ dilution) with shaking at $37^{\circ} \mathrm{C}$ for $3 \mathrm{~h}$. For strains harboring pSINA1.9, $0.1 \% \mathrm{~L}$ arabinose was supplemented to the subculture $1 \mathrm{~h}$ before harvest. Bacteria were harvested with centrifugation ( $1 \mathrm{~mL}, 6000 \times g, 1 \mathrm{~min}$, RT), washed once in $1 \times$ PBS and resuspended in DMEM with no FBS. HeLa cells were infected at a MOI of $\sim 100$ for $25 \mathrm{~min}$ at $37^{\circ} \mathrm{C}$. Extracellular bacteria were removed and washed with $1 \times$ PBS (5X). Cells were then incubated in DMEM $+10 \%$ FBS for $1 \mathrm{~h}$, washed with $1 \mathrm{x}$ PBS (3X), incubated in DMEM + 10\% FBS for $2 \mathrm{~h}$, washed with $1 \mathrm{x}$ PBS (3X) and then 
incubated in DMEM + 10\% FBS supplemented with $10 \mu \mathrm{g} / \mathrm{mL}$ gentamicin for the remaining time course of the infection.

\section{Flow cytometry}

At designated time points, cells were washed with 1 x PBS (1X) and detached with $0.05 \%$ Trypsin for $5 \mathrm{~min}$ at $37^{\circ} \mathrm{C}$. Detached cells were mixed with equal volume of DMEM $+10 \%$ FBS, passed through $40 \mu \mathrm{m}$ strainer and collected by centrifugation $\left(500 \times \mathrm{g}, 5 \mathrm{~min}, 4{ }^{\circ} \mathrm{C}\right)$. Cell pellets were dislodged and fixed in 4\% PFA (15 min, RT). Fixed cells were washed with 1 x PBS (2X) and resuspended in $200 \mu \mathrm{L} 1$ x PBS for further analysis. For digitonin permeabilization experiment, cells were permeabilized with $45 \mu \mathrm{g} / \mathrm{mL}$ digitonin ( $1 \mathrm{~min}, \mathrm{RT}$ ) or $0.25 \%$ saponin (30 min, RT), then washed and stained with anti-S. Typhimurium primary antibody and Alexa488-conjugated goat anti-rabbit secondary antibody [19]. The fluorescence intensities of the samples were assayed with LSR Fortessa (BD) (tagBFP Ex: 405 nm Em: $450 / 50$ nm; Timer ${ }^{510}$ Ex: 488 nm Em: 525/50 nm; Timer ${ }^{580}$ Ex: 562 nm Em: 582/15 nm; smURFP Ex: 633 nm Em: 670/30 nm) and analyzed with FlowJo (v10.0.4). The recorded events were gated according to the strategy described (S2 Fig).

\section{Cell sorting}

At designated time points, cells were washed with 1 x PBS (1X) and detached with $0.05 \%$ Trypsin for $5 \mathrm{~min}$ at $37^{\circ} \mathrm{C}$. Detached cells were mixed with equal volume of DMEM $+10 \%$ FBS, passed through $40 \mu \mathrm{m}$ strainer and collected by centrifugation $\left(500 \times \mathrm{g}, 5 \mathrm{~min}, 4{ }^{\circ} \mathrm{C}\right)$. Cells were washed with 1 x PBS (1X) and resuspended in DMEM $+10 \%$ FBS supplemented with $10 \mu \mathrm{g} / \mathrm{mL}$ gentamicin. Cells were sorted with Aria III (BD) (tagBFP Ex: 405 nm Em: $450 / 50$ nm; Timer ${ }^{510}$ Ex: 488 nm Em: 530/30 nm; Timer ${ }^{580}$ Ex: 561 nm Em: 586/15 nm; smURFP Ex: 633 nm Em: 660/20 nm) to collect uninfected cells, infected cells with dormant or SPI-2 S. Typhimurium populations. The recorded events were gated according to the strategy described (S2 Fig).

\section{Immunofluorescence microscopy}

Cells seeded on coverslips were washed with $1 \times$ PBS (1X) and fixed in 4\% PFA (8 min, RT). After washing with $1 \times$ PBS (3X), cells were permeabilized and blocked in 1 x PBS, 20\% FBS, $0.25 \%$ saponin (30 min, RT). Coverslips were washed with $1 \mathrm{x}$ 
PBS (3X) and incubated with anti-LC3 or anti-LAMP1 primary antibodies and phalloidin-rhodamine diluted in 1 x PBS, 2\% FBS (60 min, RT), and then washed with $1 \times$ PBS $(3 \mathrm{X})$ and incubated with Cy5-conjugated goat anti-rabbit secondary antibodies diluted in 1 x PBS, 2\% FBS (60 min, RT). Stained coverslips were then washed with $1 \mathrm{x}$ PBS (3X) and mounted on SuperFrost Plus microscope sides (Thermo Scientific) with ProLong ${ }^{\mathrm{TM}}$ Gold Antifade Mountant without DAPI (Invitrogen). Samples were imaged with Perkin Elmer Ultraview confocal spinning disk microscope equipped with Volocity software and a 20X/1.3 NA air objective. Images were analyzed with FIJI (NIH) and figures were prepared using Adobe Illustrator CS6.

\section{Colony forming unit plating}

Infected cells were enriched by cell sorting, where 1000 infected cells were sorted for each sample. The cells were then collected by centrifugation at $500 \mathrm{x} g$ for $5 \mathrm{~min}$, and subsequently lysed in $0.1 \%$ Triton X-100 for $5 \mathrm{~min}$ at room temperature. The lysed cells were then serially diluted and plated on LB agar plates with appropriate antibiotics.

\section{Dormant $S$. Typhimurium persistence assay}

Infected HeLa cells harboring dormant $S$. Typhimurium were enriched by cell sorting using the gate $\mathrm{Vac}^{-} \mathrm{Cyt}^{-}$, and plated on 12-wells plates in DMEM + 10\% FBS + Gen ${ }^{10}$. The medium was replaced with fresh DMEM $+10 \% \mathrm{FBS}+\mathrm{Gen}^{10}$ to avoid the growth of $S$. Typhimurium being released from dead cells. Cells were harvested at $24 \mathrm{~h}$ and $168 \mathrm{~h}$ pi for analysis and CFU plating.

\section{Ciprofloxacin survival assay}

A final concentration of $10 \mu \mathrm{g} / \mathrm{mL}$ of ciprofloxacin (CIP) were supplemented to the cell culture medium of the infected cells at $3 \mathrm{~h}$ pi. The cells were harvested at $6 \mathrm{~h}$ pi for cell sorting and CFU plating. CIP was administered at $3 \mathrm{~h} \mathrm{pi}$, which offered sufficient time for the infected population to differentiate into $\mathrm{Vac}^{-} \mathrm{Cyt}^{-}, \mathrm{Vac}^{+} \mathrm{Cyt}^{-}$and $\mathrm{Vac}^{+} \mathrm{Cyt}^{+}$for downstream cell sorting.

\section{Statistical analysis}


Unless further specified in the figure legend, data were analyzed for statistical significance with a Mann-Whitney test using Prism 8.0 (GraphPad). $P$ value of $\leq 0.05$ is considered statistically significant. $* P<0.05$, **P $P 0.01$, *** $P<0.001$, **** $P<$ 0.0001 , ns: not significant/ $P \geq 0.05$.

\section{Supplemental information}

Supplemental information including 4 tables, 10 figures and 3 live-imaging movies can be found with this article.

Table S1. S. Typhimurium strains used in this study

Table S2. Plasmids used in this study

Table S3. Primers used for molecular cloning in this study

Table S4. Antibodies used in this study

Supplementary figure 1. Construction strategy of SINA1.1.

Supplementary figure 2. Gating strategy of SINA1.1 reporter system.

Supplementary figure 3. Localization modules indicate subcellular localization of S. Typhimurium.

Supplementary figure 4. SINA1.1 performance in HeLa cells at $2 \mathrm{~h}, 4 \mathrm{~h}$ and $6 \mathrm{~h}$ pi.

Supplementary figure 5. S. Typhimurium exhibits distinct replication rates and metabolism in HeLa cells.

Supplementary figure 6. Dormant $S$. Typhimurium are observed as early as 2 h pi in HeLa cells.

Supplementary figure 7. Performance of SINA1.1 in Caco-2 cells. 
Supplementary figure 8. Performance of SINA1.1 in THP-1 cells.

Supplementary figure 9. Infected cells harboring dormant $S$. Typhimurium are viable.

Supplementary figure 10. Ectopic expression of hilA rescues the loss of invasiveness.

S1 Movie. Connected to Fig 1D: Time-lapse microscopy shows the fluorescence signal output from SINA1.1 in $\mathrm{Vac}^{+} \mathrm{Cyt}^{-}$intracellular $S$. Typhimurium population. Fluorescence output of Timer $^{\text {bac }}, \mathrm{P}_{s s a G}$ and $\mathrm{P}_{u h p T}$ from SINA1.1-harboring $S$. Typhimurium exhibiting $\mathrm{Vac}^{+} \mathrm{Cyt}^{-}$profile. Images were taken every 15 min starting from $1 \mathrm{~h}$ pi. (AVI)

S2 Movie. Connected to Fig 1D: Time-lapse microscopy shows the fluorescence signal output from SINA1.1 in $\mathrm{Vac}^{+} \mathrm{Cyt}^{-}$and $\mathrm{Vac}^{-} \mathrm{Cyt}^{+}$intracellular $S$. Typhimurium population. Fluorescence output of Timer ${ }^{\text {bac }}, \mathrm{P}_{s s a G}$ and $\mathrm{P}_{u h p T}$ from SINA1.1-harboring S. Typhimurium exhibiting $\mathrm{Vac}^{+} \mathrm{Cyt}{ }^{-}$and $\mathrm{Vac}^{-} \mathrm{Cyt}^{+}$profiles. Images were taken every 15 min starting from $1 \mathrm{~h}$ pi. (AVI)

S3 Movie. Connected to Fig 1D: Time-lapse microscopy shows the fluorescence signal output from SINA1.1 in $\mathrm{Vac}^{-} \mathrm{Cyt}^{-}$intracellular $S$. Typhimurium population.

Fluorescence output of Timer $^{\mathrm{bac}}, \mathrm{P}_{s s a G}$ and $\mathrm{P}_{\text {uhpT }}$ from SINA1.1-harboring $S$. Typhimurium exhibiting $\mathrm{Vac}^{-} \mathrm{Cyt}{ }^{-}$profile. Images were taken every 15 min starting from $1 \mathrm{~h}$ pi. (AVI)

\section{Author contributions}

C.H.L. and J.E. conceptualized the project, C.H.L. and Y.Y.C. conducted the investigation, C.H.L. and J.E. prepared and reviewed the manuscript.

\section{Acknowledgments}


We thank the members of the Dynamics of Host-Pathogen Interactions Unit for the constructive comment and discussion. We are grateful for the generous plasmid gift from D. Bumann. This research was supported by fellowships from Croucher Foundation (HK) and Fondation pour la Recherche Médicale (FRM) to C.H.L. and Y.Y.C.. C.H.L. is part of the Pasteur - Paris University (PPU) International PhD Program. J.E. is supported by the ERC-CoG "Endosubvert". The Enninga lab is part of the LabEx IBEID and Milieu Interieure. 


\section{References}

1. Stanaway JD, Parisi A, Sarkar K, Blacker BF, Reiner RC, Hay SI, et al. The global burden of non-typhoidal salmonella invasive disease: a systematic analysis for the Global Burden of Disease Study 2017. Lancet Infect Dis. 2019;19: 1312-1324. doi:10.1016/S1473-3099(19)30418-9

2. Shekhar C. International Conference on Food Security and Sustainable Agriculture Global impact of salmonellosis on health and economy. $\sim 93 \sim \mathrm{J}$ Pharmacogn Phytochem. 2018;4.

3. Ilyas B, Tsai CN, Coombes BK. Evolution of Salmonella-host cell interactions through a dynamic bacterial genome. Frontiers in Cellular and Infection Microbiology. Frontiers Media S.A.; 2017. p. 428. doi:10.3389/fcimb.2017.00428

4. Lou L, Zhang P, Piao R, Wang Y. Salmonella Pathogenicity Island 1 (SPI-1) and Its Complex Regulatory Network. Frontiers in Cellular and Infection Microbiology. Frontiers Media S.A.; 2019. p. 270. doi:10.3389/fcimb.2019.00270

5. Jennings E, Thurston TLM, Holden DW. Salmonella SPI-2 Type III Secretion System Effectors: Molecular Mechanisms And Physiological Consequences. Cell Host Microbe. 2017;22: 217-231. doi:10.1016/J.CHOM.2017.07.009

6. LaRock DL, Chaudhary A, Miller SI. S. Typhimurium interactions with host processes. Nat Rev Microbiol. 2015;13: 191-205. doi:10.1038/nrmicro3420

7. Knodler LA. Salmonella enterica: Living a double life in epithelial cells. Curr Opin Microbiol. 2015;23: 23-31. doi:10.1016/j.mib.2014.10.010

8. Stévenin V, Chang YY, Le Toquin Y, Duchateau M, Gianetto QG, Luk CH, et al. Dynamic Growth and Shrinkage of the Salmonella-Containing Vacuole Determines the Intracellular Pathogen Niche. Cell Rep. 2019;29: 3958-3973.e7. doi:10.1016/j.celrep.2019.11.049

9. Fredlund J, Santos JC, Stévenin V, Weiner A, Latour-Lambert P, Rechav K, et al. The entry of Salmonella in a distinct tight compartment revealed at high temporal and ultrastructural resolution. Cell Microbiol. 2018;20: e12816. doi:10.1111/cmi.12816

10. López-Montero N, Ramos-Marquès E, Risco C, García-del Portillo F. Intracellular Salmonella induces aggrephagy of host endomembranes in persistent infections. Autophagy. 2016;12: 1886-1901. 
doi:10.1080/15548627.2016.1208888

11. Helaine S, Thompson $\mathrm{J}$ a., Watson KG, Liu M, Boyle C, Holden DW. Dynamics of Intracellular Bacterial Replication at the Single Cell Level. Proc Natl Acad Sci. 2010;107: 3746-3751. doi:10.1073/pnas.1000041107

12. Helaine S, Cheverton AM, Watson KG, Faure LM, Matthews SA, Holden DW. Internalization of salmonella by macrophages induces formation of nonreplicating persisters. Science (80- ). 2014;343: 204-208. doi:10.1126/science. 1244705

13. Lewis K. Persister Cells. 2010 [cited 15 Feb 2019]. doi:10.1146/annurev.micro.112408.134306

14. Cheverton AM, Gollan B, Przydacz M, Wong CT, Mylona A, Hare SA, et al. A Salmonella Toxin Promotes Persister Formation through Acetylation of tRNA. Mol Cell. 2016;63: 86-96. doi:10.1016/j.molcel.2016.05.002

15. Bakkeren E, Huisman JS, Fattinger SA, Hausmann A, Furter M, Egli A, et al. Salmonella persisters promote the spread of antibiotic resistance plasmids in the gut. Nature. 2019; 1-5. doi:10.1038/s41586-019-1521-8

16. Spinnenhirn V, Farhan H, Basler M, Aichem A, Canaan A, Groettrup M. The ubiquitin-like modifier FAT10 decorates autophagy-targeted Salmonella and contributes to Salmonella resistance in mice. J Cell Sci. 2014;127: 4883-93. doi:10.1242/jcs.152371

17. Hapfelmeier S, Stecher B, Barthel M, Kremer M, Müller AJ, Heikenwalder M, et al. The Salmonella Pathogenicity Island (SPI)-2 and SPI-1 Type III Secretion Systems Allow Salmonella Serovar typhimurium to Trigger Colitis via MyD88-Dependent and MyD88-Independent Mechanisms . J Immunol. 2005;174: 1675-1685. doi:10.4049/jimmunol.174.3.1675

18. Claudi B, Spröte P, Chirkova A, Personnic N, Zankl J, Schürmann N, et al. Phenotypic variation of salmonella in host tissues delays eradication by antimicrobial chemotherapy. Cell. 2014;158: 722-733. doi:10.1016/j.cell.2014.06.045

19. Knodler LA, Nair V, Steele-Mortimer O. Quantitative assessment of cytosolic Salmonella in epithelial cells. PLoS One. 2014;9: e84681. doi:10.1371/journal.pone.0084681

20. Lau N, Haeberle AL, O’Keeffe BJ, Latomanski EA, Celli J, Newton HJ, et al. SopF, a phosphoinositide binding effector, promotes the stability of the nascent 
Salmonella-containing vacuole. PLoS Pathog. 2019;15: e1007959. doi:10.1371/journal.ppat.1007959

21. Knodler LA, Vallance BA, Celli J, Winfree S, Hansen B, Montero M, et al. Dissemination of invasive Salmonella via bacterial-induced extrusion of mucosal epithelia. Proc Natl Acad Sci U S A. 2010;107: 17733-17738. doi:10.1073/pnas.1006098107

22. Yu HB, Croxen MA, Marchiando AM, Ferreira RBR, Cadwell K, Foster LJ, et al. Autophagy Facilitates Salmonella Replication in HeLa Cells. MBio. 2014;5: e00865-14. doi:10.1128/MBIO.00865-14

23. Birmingham CL, Smith AC, Bakowski MA, Yoshimori T, Brumell JH. Autophagy controls Salmonella infection in response to damage to the Salmonella-containing vacuole. J Biol Chem. 2006;281: 11374-11383. doi:10.1074/jbc.M509157200

24. Terskikh a, Fradkov A, Ermakova G, Zaraisky A, Tan P, Kajava a V, et al. "Fluorescent timer": protein that changes color with time. Science. 2000;290: 1585-1588. doi:10.1126/science.290.5496.1585

25. Cunrath O, Bumann D. Host resistance factor SLC11A1 restricts Salmonella growth through magnesium deprivation. Science (80- ). 2019;366: 995-999. doi:10.1126/science.aax7898

26. Steele-Mortimer O. The Salmonella-containing vacuole-Moving with the times. Current Opinion in Microbiology. NIH Public Access; 2008. pp. 38-45. doi:10.1016/j.mib.2008.01.002

27. Stapels DAC, Hill PWS, Westermann AJ, Fisher RA, Thurston TL, Saliba A-E, et al. Salmonella persisters undermine host immune defenses during antibiotic treatment. Science $\quad(80-\quad)$ 2018;362: 1156-1160. doi:10.1126/SCIENCE.AAT7148

28. Santos JC, Enninga J. At the crossroads: communication of bacteria $\square$ containing vacuoles with host organelles. Cell Microbiol. 2016;18: 330-339. doi:10.1111/cmi.12567

29. Shea JE, Hensel M, Gleeson C, Holden DW. Identification of a virulence locus encoding a second type III secretion system in Salmonella typhimurium. Proc Natl Acad Sci U S A. 1996;93: 2593-2597. doi:10.1073/pnas.93.6.2593

30. Diard M, Hardt W-D. Basic Processes in Salmonella-Host Interactions: WithinHost Evolution and the Transmission of the Virulent Genotype. Microbiol 
Spectr. 2017;5: 1-11. doi:10.1128/microbiolspec.mtbp-0012-2016

31. Hindle Z, Chatfield SN, Phillimore J, Bentley M, Johnson J, Cosgrove CA, et al. Characterization of Salmonella enterica derivatives harboring defined aroC and Salmonella pathogenicity island 2 type III secretion system (ssaV) mutations by immunization of healthy volunteers. Infect Immun. 2002;70: 3457-3467. doi:10.1128/IAI.70.7.3457-3467.2002

32. Dalebroux ZD, Svensson SL, Gaynor EC, Swanson MS. ppGpp Conjures Bacterial Virulence. Microbiol Mol Biol Rev. 2010;74: 171-199. doi:10.1128/mmbr.00046-09

33. Dalebroux ZD, Swanson MS. PpGpp: Magic beyond RNA polymerase. Nature Reviews Microbiology. 2012. pp. 203-212. doi:10.1038/nrmicro2720

34. Helaine S, Kugelberg E. Bacterial persisters: formation, eradication, and experimental systems. Trends Microbiol. 2014;22: 417-424. doi:10.1016/J.TIM.2014.03.008

35. Pizarro-Cerdá J, Tedin K. The bacterial signal molecule, ppGpp, regulates Salmonella virulence gene expression. Mol Microbiol. 2004;52: 1827-1844. doi:10.1111/j.1365-2958.2004.04122.x

36. Hauryliuk V, Atkinson GC, Murakami KS, Tenson T, Gerdes K. Recent functional insights into the role of (p)ppGpp in bacterial physiology. Nature Reviews Microbiology. Nature Publishing Group; 2015. pp. 298-309. doi:10.1038/nrmicro3448

37. Gaca AO, Colomer-Winter C, Lemos JA. Many means to a common end: the intricacies of (p)ppGpp metabolism and its control of bacterial homeostasis. J Bacteriol. 2015;197: 1146-56. doi:10.1128/JB.02577-14

38. Hobbs JK, Boraston AB. (p)ppGpp and the Stringent Response: An Emerging Threat to Antibiotic Therapy. 2019 [cited 1 Aug 2019]. doi:10.1021/acsinfecdis.9b00204

39. Maisonneuve E, Gerdes K. Molecular Mechanisms Underlying Bacterial Persisters. Cell. 2014;157: 539-548. doi:10.1016/J.CELL.2014.02.050

40. Diard M, Bakkeren E, Cornuault JK, Moor K, Hausmann A, Sellin ME, et al. Inflammation boosts bacteriophage transfer between Salmonella spp. Science (80- ). 2017;355: 1211-1215. doi:10.1126/science.aaf8451

41. Santiviago CA, Reynolds MM, Porwollik S, Choi S-H, Long F, AndrewsPolymenis HL, et al. Analysis of Pools of Targeted Salmonella Deletion 
Mutants Identifies Novel Genes Affecting Fitness during Competitive Infection in Mice. Cookson BT, editor. PLoS Pathog. 2009;5: e1000477. doi:10.1371/journal.ppat.1000477

42. Tanenbaum ME, Gilbert LA, Qi LS, Weissman JS, Vale RD. A protein-tagging system for signal amplification in gene expression and fluorescence imaging. Cell. 2014;159: 635-646. doi:10.1016/j.cell.2014.09.039

43. Campbell-Valois FX, Schnupf P, Nigro G, Sachse M, Sansonetti PJ, Parsot C. A fluorescent reporter reveals on/off regulation of the shigella type III secretion apparatus during entry and cell-to-cell spread. Cell Host Microbe. 2014;15: 177-189. doi:10.1016/j.chom.2014.01.005

44. Rodriguez EA, Tran GN, Gross LA, Crisp JL, Shu X, Lin JY, et al. A far-red fluorescent protein evolved from a cyanobacterial phycobiliprotein. Nat Methods. 2016;13: 763-769. doi:10.1038/nmeth.3935 

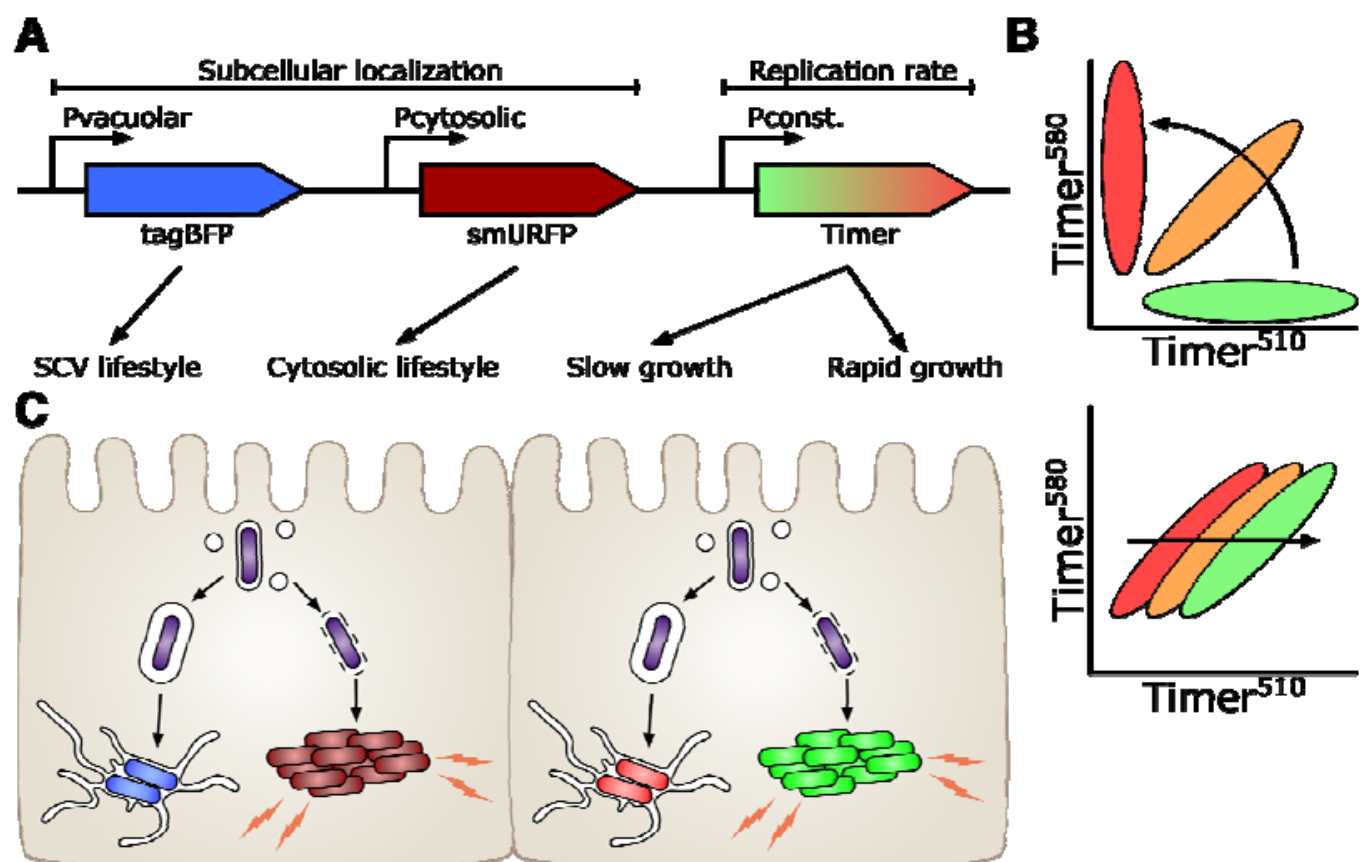

D
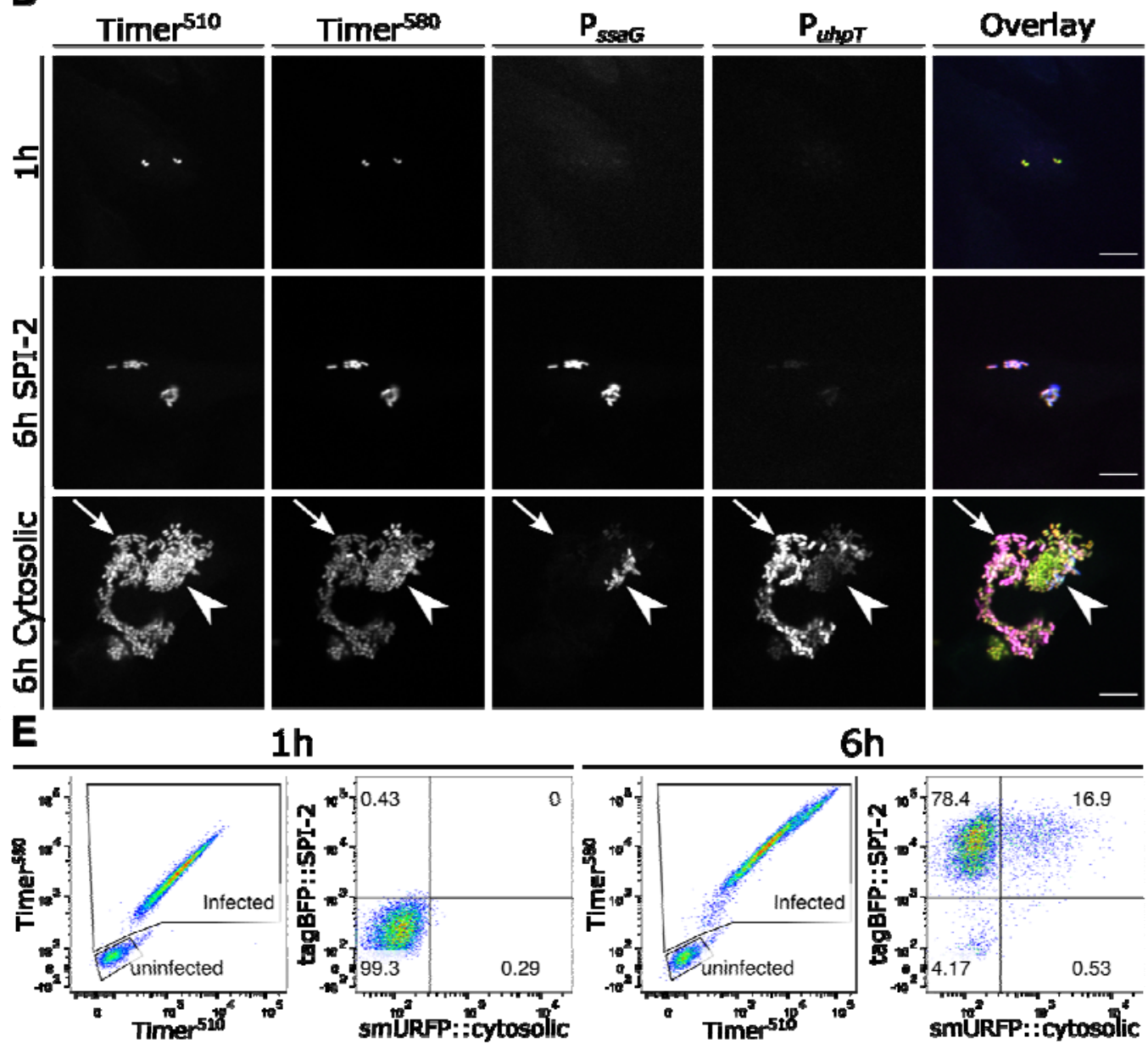

1h
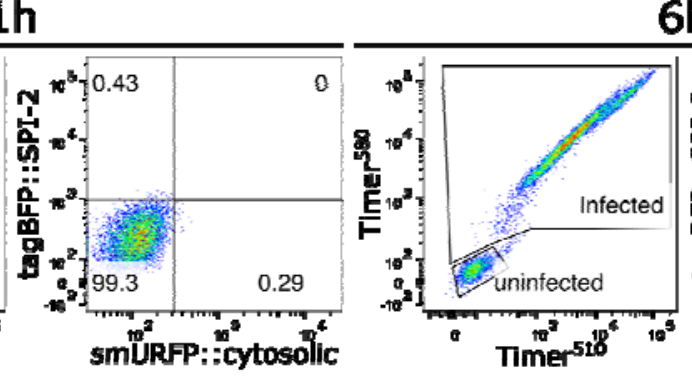

6h

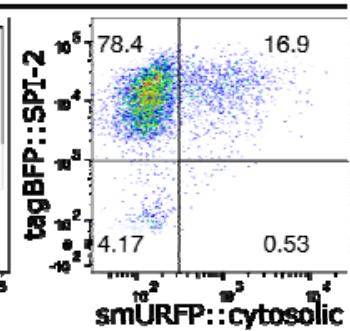

Figure 1. SINA enables precise determination of the different Salmonella intracellular lifestyles in human epithelial cells. 
(A) Schematic diagram of the construction of subcellular localization and replication rate modules of SINA1.1. The subcellular localization module is composed of the vacuolar submodule $\left(\mathrm{P}_{s s a G}\right.$-tagBFP) and cytosolic submodule $\left(\mathrm{P}_{u h p T}\right.$-smURFP), while the replication rate module is composed of a constitutively expressed $\operatorname{Timer}^{\mathrm{bac}}\left(\mathrm{P}_{y b a J^{-}}\right.$ Timer $^{\text {bac }}$ ) (B) (Top) Schematic diagram of the emission spectrum shifts of $S$. Typhimurium harboring Timer ${ }^{\text {bac }}$ as Timer $^{\text {bac }}$ matures, where emission shifts from green to red (Bottom) Green:Red ratio increases with elevating S. Typhimurium replication rates. As $S$. Typhimurium divides, both $\operatorname{Timer}^{510}$ and $\operatorname{Timer}^{580}$ fluorophores are diluted. With a higher production rate of $\operatorname{Timer}^{510}$ than $\operatorname{Timer}^{580}$, fast dividing $S$. Typhimurium exhibits a higher Green:Red ratio. (C) Expected output by SINA as $S$. Typhimurium dwells in distinct subcellular localizations. Vacuolar $S$. Typhimurium are of lower replication rate (i.e. lower Green:Red ratio) and are expected to emit blue fluorescence; cytosolic $S$. Typhimurium are of higher replication rate (i.e. higher Green:Red ratio) and are expected to emit far red fluorescence (D) HeLa cells infected by $S$. Typhimurium harboring SINA1.1. Output of SINA from intracellular $S$. Typhimurium was detected by fluorescence microscopy at $1 \mathrm{~h}$ pi, vacuolar (arrowhead) and cytosolic (arrow) $S$. Typhimurium at $6 \mathrm{~h}$ pi. (3 independent experiments). Scale bar is $10 \mu \mathrm{m}$. (E) HeLa cells infected by $S$. Typhimurium harboring SINA1.1. Output of SINA from intracellular $S$. Typhimurium at $1 \mathrm{~h}$ and $6 \mathrm{~h}$ pi was detected by flow cytometry ( 3 independent experiments). 
A

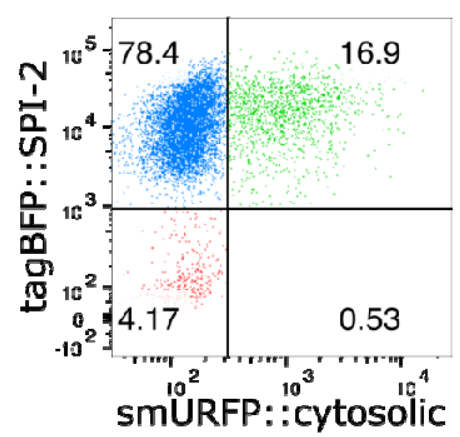

B

SINA1.4

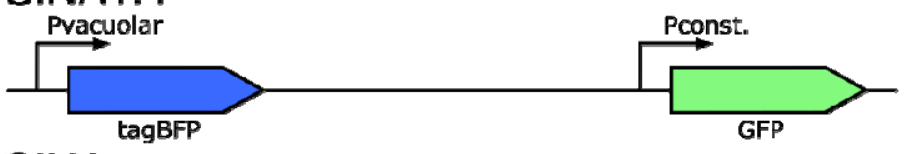

SINA1.5

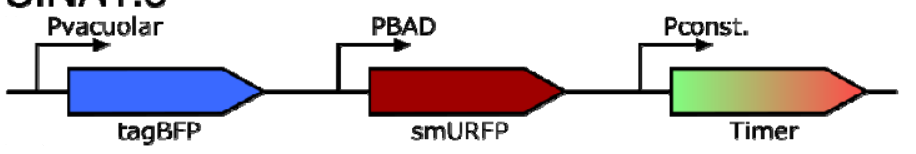

C

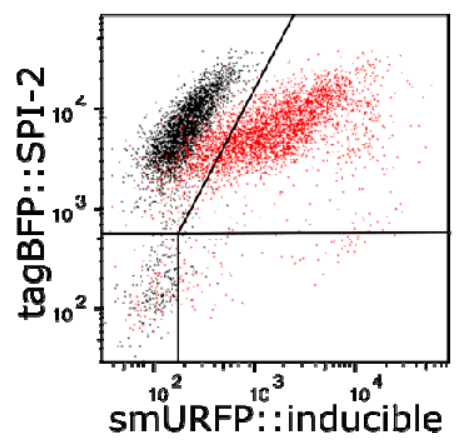

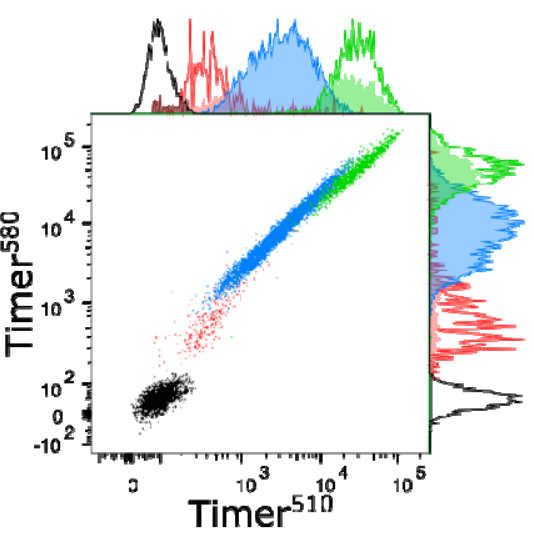

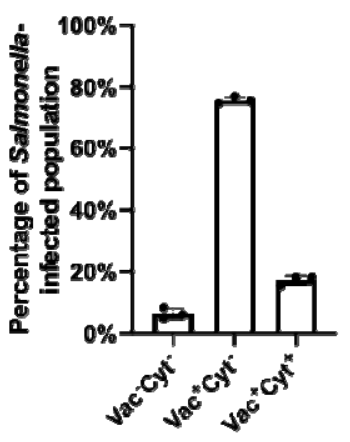

D

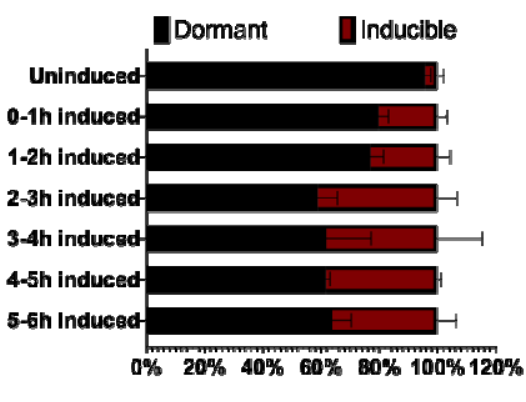

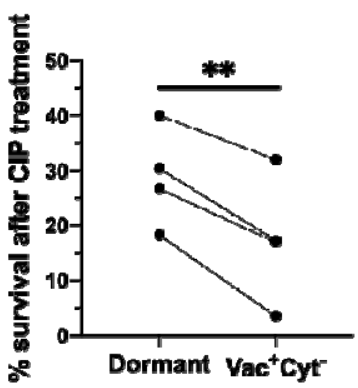

Figure 2. $S$. Typhimurium displays a novel dormant intracellular lifestyle in epithelial cells.

(A) (Left and Middle) Timer ${ }^{\text {bac }}$ profile and distribution of single cells with no infection ( $\left.\mathrm{Vac}^{-} \mathrm{Cyt}{ }^{-}\right)$(red), infected cells with only vacuolar bacteria $\left(\mathrm{Vac}^{+} \mathrm{Cyt} \mathrm{t}^{-}\right)$(blue) and infected cells with both vacuolar and cytosolic populations $\left(\mathrm{Vac}^{+} \mathrm{Cyt}^{+}\right)$(green) at 6 h pi. (Right) Abundance of $S$. Typhimurium-infected cells ( $\mathrm{Vac}^{-} \mathrm{Cyt}^{-}, \mathrm{Vac}^{+} \mathrm{Cyt}^{-}$and $\left.\mathrm{Vac}^{+} \mathrm{Cyt}^{+}\right)$as illustrated in (A) $(\mathrm{n}=3)$. (B) Schematic illustration for the constructions of SINA derivatives, SINA1.4 and SINA1.5. SINA1.4 was used for immunofluorescence staining against LAMP1 and LC3; SINA1.5 was used for arabinose induction assay. (C) (Left) Responsiveness of intracellular $S$. Typhimurium towards an arabinose pulse between 5-6 h pi, uninduced control (black); arabinoseinduced (red). (Right) Quantification on the responsiveness of $\mathrm{Vac}^{-} S$. Typhimurium 
pulsed at different time intervals during the infection time course, dormant (black), inducible (maroon). Samples were all harvested at $6 \mathrm{~h}$ pi. $(\mathrm{n}=3)(\mathrm{D})$ Survival percentage of dormant and $\mathrm{Vac}^{+} \mathrm{Cyt}^{-}$intracellular $S$. Typhimurium against $3 \mathrm{~h}$ of CIP treatment, infected cells were harvested at $6 \mathrm{~h}$ pi, enriched by cell sorting and plated for CFU. $(n=3)$ 

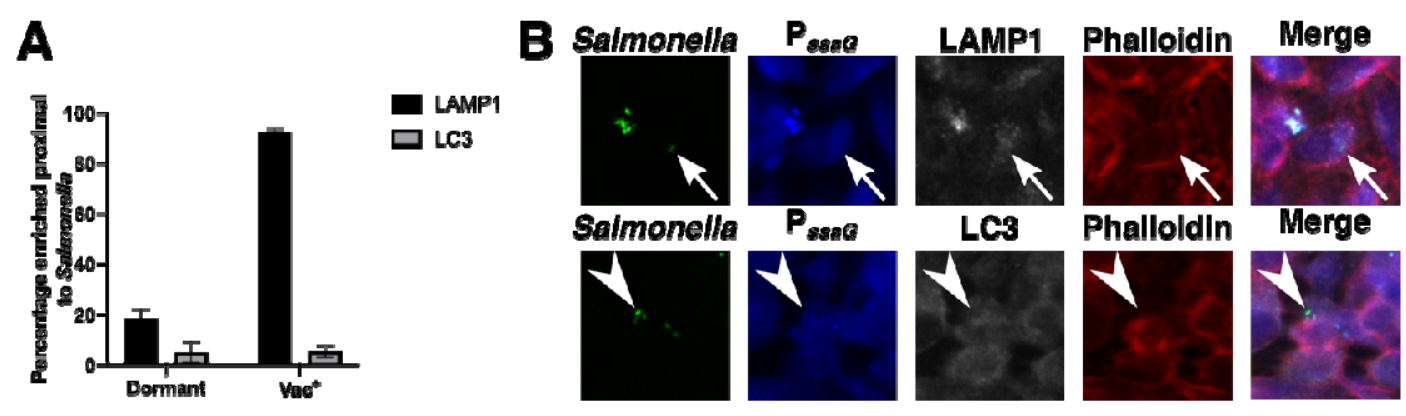

Figure 3. Dormant $S$. Typhimurium dwells in a vacuolar compartment distinct from the conventional $S C V$.

(A) HeLa cells were infected with SINA1.4-harboring S. Typhimurium, harvested at 6 $\mathrm{h}$ pi, fixed and stained. Quantification of the presence of LAMP1 and LC3 proximal to $\mathrm{Vac}^{-} \mathrm{Cyt}^{-}$and $\mathrm{Vac}^{+} S$. Typhimurium at $6 \mathrm{~h}$ pi. $(\mathrm{n}=3)(\mathrm{B})$ Representative images of $\operatorname{Vac}^{-} S$. Typhimurium quantified in (A); $S$. Typhimurium (green), $\mathrm{Vac}^{-}$(blue), LAMP1 (grey, top), LC3 (grey, bottom), Phalloidin (red). 

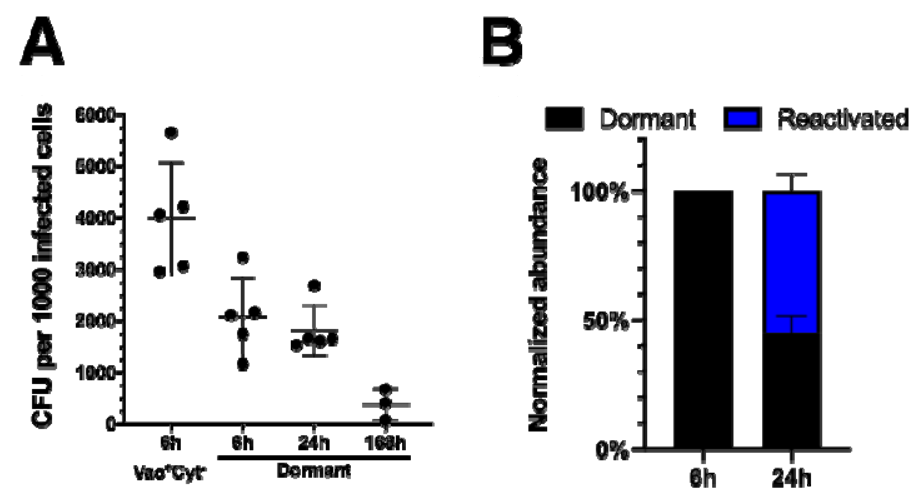

Figure 4. Dormant $S$. Typhimurium persists in host cells and reactivates virulence gene expression.

(A) Designated populations of infected cells were enriched by cell sorting and plated for CFU. Quantification of CFU from dormant $S$. Typhimurium at $6 \mathrm{~h}, 24 \mathrm{~h}$ and $168 \mathrm{~h}$ pi and $\mathrm{Vac}^{+} \mathrm{Cyt}^{-} S$. Typhimurium at $6 \mathrm{~h}$ pi. (n $=5$ for $\operatorname{Vac}^{+} \mathrm{Cyt}{ }^{-} 6 \mathrm{~h}$, Dormant $6 \mathrm{~h}, 24 \mathrm{~h}$; $\mathrm{n}=3$ for Dormant 168 h) (B) Quantification of SPI-2 activity using flow cytometry in enriched dormant $S$. Typhimurium at $6 \mathrm{~h}$ and enriched $\mathrm{Vac}^{-} \mathrm{Cyt}^{-}$infected cells replated until 24 h pi. $(n=3)$ 

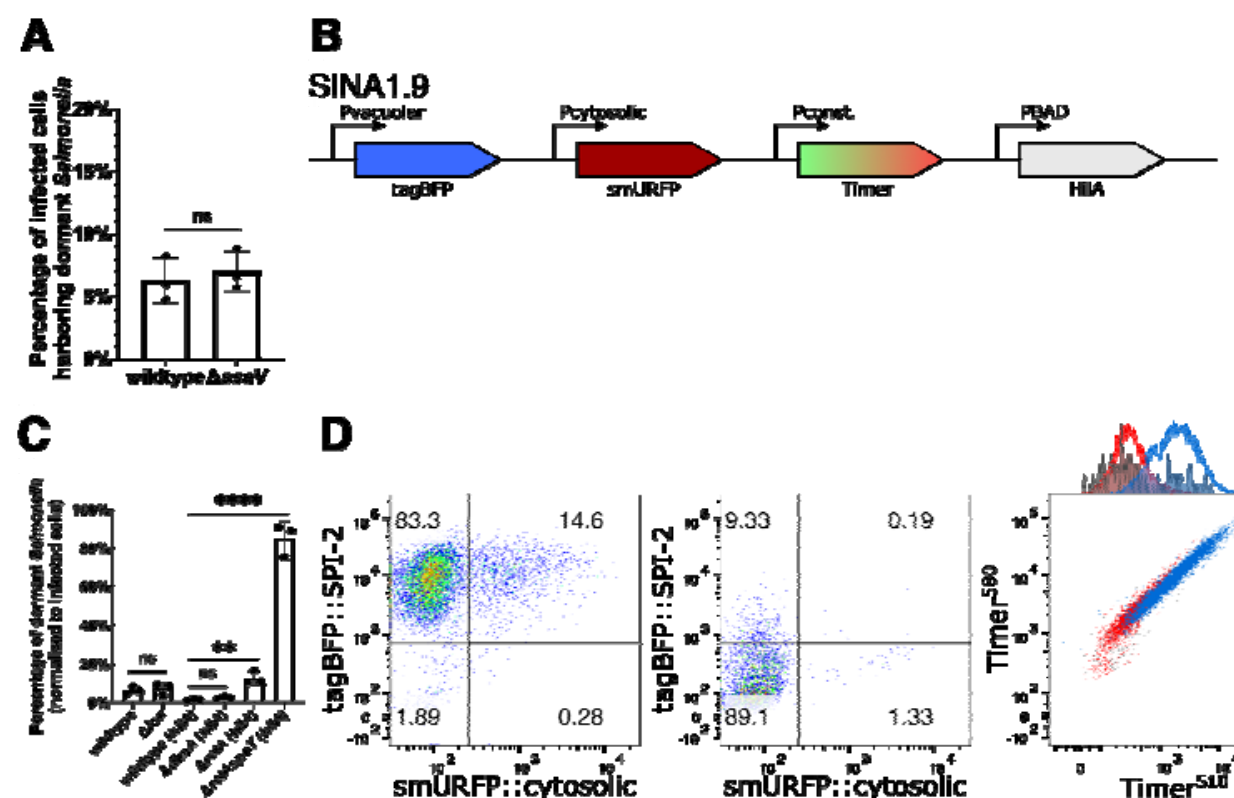

D
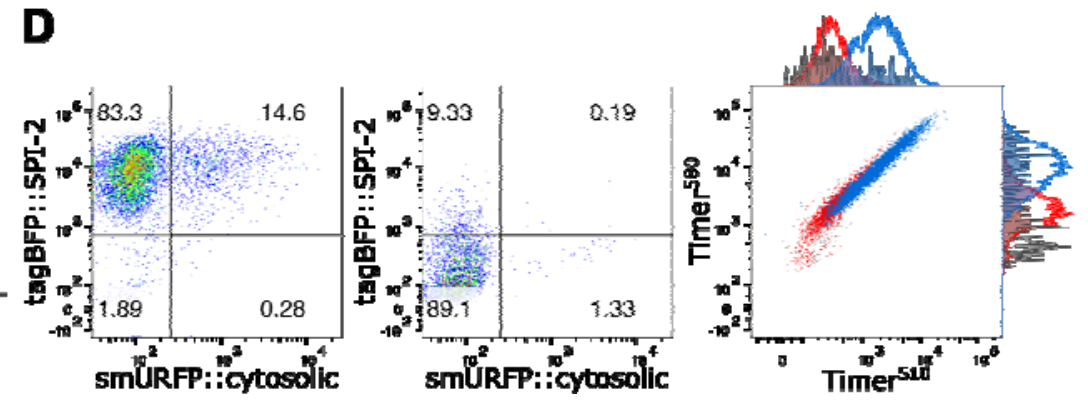

Figure 5. S. Typhimurium dormancy is negatively regulated by SpoT.

(A) HeLa cells were infected with SINA1.1-harboring $S$. Typhimurium, the abundance of $\mathrm{Vac}^{-} \mathrm{Cyt}^{-}$population in wild type and SPI-2 mutant $\Delta s s a V$ infected cells were quantified with flow cytometry at $6 \mathrm{~h}$ pi. $(\mathrm{n}=3)(\mathrm{B})$ Schematic diagram for the construction of SINA derivative, SINA1.9, yielded from the introduction of an arabinose-inducible hilA expression cassette into SINA1.1. SINA1.9 was used to rescue the reduced invasiveness of $\triangle d k s A, \triangle r e l A$ and $\triangle$ relAspoT mutant strains. (C) HeLa cells were infected with SINA1.1 or SINA1.9-harboring S. Typhimurium, the abundance of $\mathrm{Vac}^{-} \mathrm{Cyt}^{-}$population in (p)ppGpp biogenesis and regulon mutants, Alon, $\triangle d k s A, \triangle r e l A$ and $\triangle r e l A s p o T$ were quantified by flow cytometry at $6 \mathrm{~h}$ pi $(\mathrm{n}=3)(\mathrm{D})$ Distribution of $\mathrm{Vac}^{-} \mathrm{Cyt}{ }^{-}, \mathrm{Vac}^{+} \mathrm{Cyt}^{-}$and $\mathrm{Vac}^{+} \mathrm{Cyt}^{+}$populations in wild type (Left) and ArelAspoT mutant (Middle) infected HeLa cells at $6 \mathrm{~h}$ pi quantified by flow cytometry. Overlay Timer ${ }^{\text {bac }}$ profile (Left) of $\mathrm{Vac}^{-} \mathrm{Cyt}^{-}$(red) and $\mathrm{Vac}^{+} \mathrm{Cyt}^{-}$(blue) populations of wild type and $\mathrm{Vac}^{-} \mathrm{Cyt}^{-}$population of ArelAspoT mutant (grey) in infected HeLa cells quantified by flow cytometry at $6 \mathrm{~h}$ pi. (3 independent experiments) Statistics were performed using unpaired t test. ns: not significant $(P>0.05), * * P<0.01, * * * * P<$ 0.0001 . 


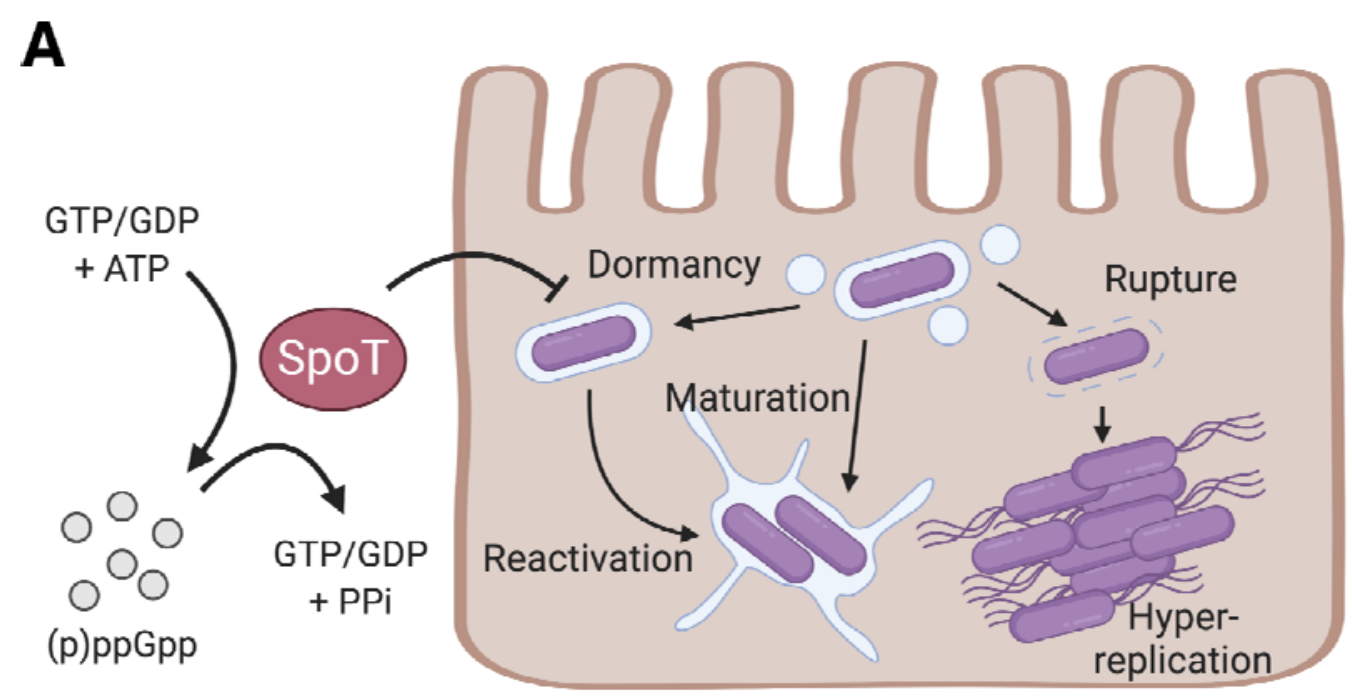

B

\section{Progression of infection}

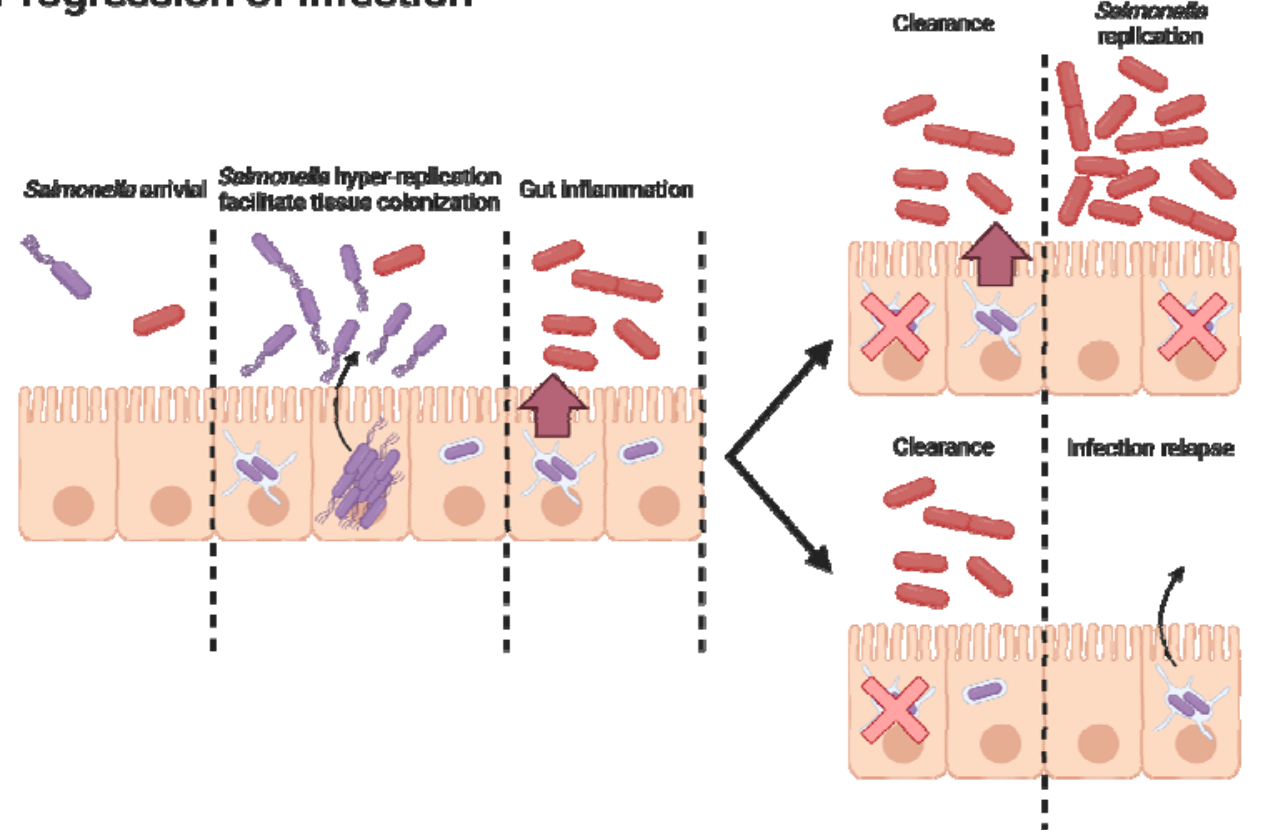

Figure 6. Schematic illustration of the role of (p)ppGpp alarmone pathway on $S$.

Typhimurium dormancy in enterocytes and the proposed pathophysiological implication of $S$. Typhimurium dormancy in enterocytes. (A) Schematic diagram of $S$. Typhimurium lifestyles and the regulatory role of SpoT on $S$. Typhimurium dormancy in human epithelial cells. $S$. Typhimurium can opt for three distinct lifestyles: cytosolic, vacuolar and dormant, which exhibits discernible subcellular localization, replication rate and metabolism. The entry of dormant state is negatively regulated by (p)ppGpp synthatase SpoT, while the regulatory mechanism on the 
dormancy exit remains to be determined. (B) Schematic diagram of $S$. Typhimurium infection progression in the gut epithelium. As $S$. Typhimurium reaches the intestinal epithelium, a portion of $S$. Typhimurium expresses T3SS1 (purple) to enter host cells and adopts various intracellular lifestyles. Distinct $S$. Typhimurium lifestyles support rapid tissue colonization and gut inflammation to increase competitiveness of luminal $S$. Typhimurium (red). (Top) Reactivation of dormant $S$. Typhimurium leads to prolonged gut inflammation that supports the continuous growth of $S$. Typhimurium at gut lumen. (Bottom) Dormant $S$. Typhimurium reactivates after the eradication of gut $S$. Typhimurium, which serves as the reservoirs of infection relapse. 


\section{Supporting Information}

\section{Salmonella endorses a dormant state within human epithelial cells for persistent infection}

Chak Hon Luk ${ }^{1,2}$, Yuen-Yan Chang ${ }^{1}$, Jost Enninga ${ }^{1,2 *}$

${ }^{1}$ Dynamics of Host-Pathogen Interactions Unit, Institut Pasteur, 75724 Paris, France

${ }^{2}$ Université de Paris, Sorbonne Paris Cité, Paris, France

*Corresponding author. Email: jost.enninga@pasteur.fr (J.E.) 
Table S1. S. Typhimurium strains used in this study

\begin{tabular}{llll}
\hline Name & Genotype & Plasmid & Reference \\
\hline JL129 & Wild type & pSINA1.1 & This study \\
JL147 & Wild type & pSINA1.4 & This study \\
JL148 & Wild type & pSINA1.5 & This study \\
JL177 & Wild type & pSINA1.7 & This study \\
JL179 & Wild type & pSINA1.9 & This study \\
JL171 & AssrB & pSINA1.1 & This study \\
JL173 & AssaV & pSINA1.1 & This study \\
JL158 & Alon & pSINA1.1 & This study \\
JL180 & AdksA & pSINA1.9 & This study \\
JL185 & ArelA & pSINA1.9 & This study \\
JL181 & ArelA AspoT & pSINA1.9 & This study \\
\hline
\end{tabular}


Table S2. Plasmids used in this study

\begin{tabular}{|c|c|c|}
\hline Name & Description & Reference \\
\hline $\begin{array}{l}\text { pBR322 } \\
\text { Timer }^{\text {bac }}\end{array}$ & Timer $^{\text {bac }}$ expression & Claudi et al. 2014 \\
\hline pM973 & SPI-2 activity of $S$. Typhimurium & Hapfelmeier et al. 2005 \\
\hline pTSAR1 & Shigella T3SS activity & $\begin{array}{l}\text { Campbell-Valois et al. } \\
2014\end{array}$ \\
\hline $\begin{array}{l}\text { pHRdSV40- } \\
\text { NLS-dCas9- }\end{array}$ & & \\
\hline $\begin{array}{l}\text { 24xGCN4_v4- } \\
\text { NLS-P2A-BFP- } \\
\text { dWPRE }\end{array}$ & Template of tagBFP & Tanenbaum et al. 2014 \\
\hline $\begin{array}{l}\text { pBAD smURFP- } \\
\text { HO-1 }\end{array}$ & Inducible expression of smURFP & Rodriguez et al. 2016 \\
\hline pPssaG-tagBFP & SPI-2 activity of $S$. Typhimurium & This study \\
\hline $\begin{array}{l}\text { pPuhpT- } \\
\text { smURFP }\end{array}$ & Cytosolic access of $S$. Typhimurium & This study \\
\hline pSINA-int & $\begin{array}{l}\text { Subcloning PssaG-tagBFP and } \\
\text { PuhpT-smURFP }\end{array}$ & This study \\
\hline pSINA1.1 & $\begin{array}{l}\text { Detection of } S . \quad \text { Typhimurium } \\
\text { lifestyles }\end{array}$ & This study \\
\hline pSINA1.4 & $\begin{array}{l}\text { Detection of } S . \quad \text { Typhimurium } \\
\text { lifestyles using immunofluorescence }\end{array}$ & This study \\
\hline pSINA1.5 & $\begin{array}{l}\text { Detection of } S \text {. Typhimurium } \\
\text { lifestyles with inducible smURFP } \\
\text { expression }\end{array}$ & This study \\
\hline
\end{tabular}




$\begin{array}{lllllll}\text { pSINA1.7 } & \text { Detection } & \text { of } & S . & \text { Typhimurium } & \\ & \text { lifestyles } & & & & & \text { This study } \\ \text { pBAD hilA } & \text { Inducible expression of } & & & \\ & \text { Detection } & \text { of } & S . & \text { Typhimurium } & \\ \text { pSINA1.9 } & \text { lifestyles } & \text { with } & \text { inducible } & \text { hild } & \text { This study } \\ & \text { expression } & & & & & \\ & & & & & & \end{array}$

Table S3. Primers used for molecular cloning in this study

\begin{tabular}{|c|c|c|}
\hline Name & Sequence & Purpose \\
\hline tagBFP_fw & $\begin{array}{l}\text { GAATTCAGGAGGTAGTATTGatgagcgagctgattaagga } \\
\text { g }\end{array}$ & $\begin{array}{l}\text { Amplification } \\
\text { of } \operatorname{tagBFP}\end{array}$ \\
\hline tagBFP_rv & $\underline{\text { ATCttaattaagettgtgecceag }}$ & $\begin{array}{l}\text { Amplification } \\
\text { of } \operatorname{tagBFP}\end{array}$ \\
\hline & & Amplification \\
\hline uhpT_fw & GAATTCcgcgagacceacgaagcgtg & $\begin{array}{l}\text { of } \quad u h p T \\
\text { promoter }\end{array}$ \\
\hline & & Amplification \\
\hline uhpT_rv & $\underline{\text { GGATCCCATggattactcetgagc }}$ & $\begin{array}{l}\text { of } u h p T \\
\text { promoter }\end{array}$ \\
\hline smURFP_fw & ggatccAAAACTTCTGAACAACGTGTAAACATCGC & $\begin{array}{l}\text { Amplification } \\
\text { of } \operatorname{smURFP}\end{array}$ \\
\hline SmURFP_rv & tctagaCTAGCCTTCGGAGGTGGCgag & $\begin{array}{l}\text { Amplification } \\
\text { of } \operatorname{smURFP}\end{array}$ \\
\hline Vac_fw & TCTAGACGGTAGATTAGCCTTAACCGCg & $\begin{array}{l}\text { Amplification } \\
\text { of vacuolar } \\
\text { module }\end{array}$ \\
\hline Cyt_rv & gcatgcGTAAAACGACGGCCAGTGCC & $\begin{array}{l}\text { Amplification } \\
\text { of cytosolic } \\
\text { module }\end{array}$ \\
\hline GFP_fw & $\begin{array}{l}\text { tctagatttaagaaggagatatacatATGAGTAAAGGAGAAG } \\
\text { AACTTTTCACTGGA }\end{array}$ & $\begin{array}{l}\text { Amplification } \\
\text { of } G F P\end{array}$ \\
\hline
\end{tabular}




\section{GFP_rv AAGCTTTTATTTGTATAGTTCATCCATGCC}

Ara_fw GTCGACatgtgectgtcaaatggacg

Ara_rv

GCATGCGTAGAAACGCAAAAAGGCCATCCG

DsRed_fw gtcacgtagtagtagccgggc

DsRed_rv tccaagetggacatcacetcce

hilA_fw1 GGATCCatgecacattttaatcetgttce

hilA_rv1

gtttAAACttaccgtaatttaatcaagcggggGtcctgtttccatcttttgaa

cc

hilA_fw2 atctaaGTCGACatgtgectgtcaaatggacg

hilA_rv2 gtagaaacgcaaaaaggccatccg
Amplification

of $G F P$

Amplification

of inducible

SMURFP

cassette

Amplification

of inducible

SMURFP

cassette

Mutagenesis

of Timer $^{b a c}$ to

DsRed

Mutagenesis

of Timer $^{\text {bac }}$ to

DsRed

Amplification

of hilA

Amplification

of hilA

Amplification

of inducible

hilA cassette

Amplification

of inducible

hilA cassette 
Table S4. Antibodies used in this study

\begin{tabular}{|c|c|c|c|}
\hline Name of reagent & Concentration & Supplier & Reference \\
\hline $\begin{array}{l}\text { Anti-LAMP1 } \\
\text { antibody }\end{array}$ & $1: 200$ & abcam & ab19294 \\
\hline Anti-LC3B antibody & $1: 200$ & abcam & ab51520 \\
\hline $\begin{array}{l}\text { Anti-S. Typhimurium } \\
\text { antibody }\end{array}$ & $1: 200$ & abcam & ab35156 \\
\hline $\begin{array}{l}\text { Goat anti-Rabbit IgG } \\
(\mathrm{H}+\mathrm{L}) \text { Highly Cross- }\end{array}$ & & & \\
\hline $\begin{array}{lr}\text { Adsorbed } & \text { Secondary } \\
\text { Antibody, } & \text { Alexa } \\
\text { Fluor } 488 & \end{array}$ & $1: 200$ & Invitrogen & A11034 \\
\hline Goat anti-Rabbit IgG & & & \\
\hline $\begin{array}{lr}(\mathrm{H}+\mathrm{L}) & \text { Cross- } \\
\text { Adsorbed } & \text { Secondary }\end{array}$ & $1: 200$ & Invitrogen & A10523 \\
\hline Antibody, Cyanine5 & & & \\
\hline $\begin{array}{l}\text { Rhodamine } \\
\text { Phalloidin }\end{array}$ & $1: 100$ & Invitrogen & $\mathbf{R 4 1 5}$ \\
\hline
\end{tabular}




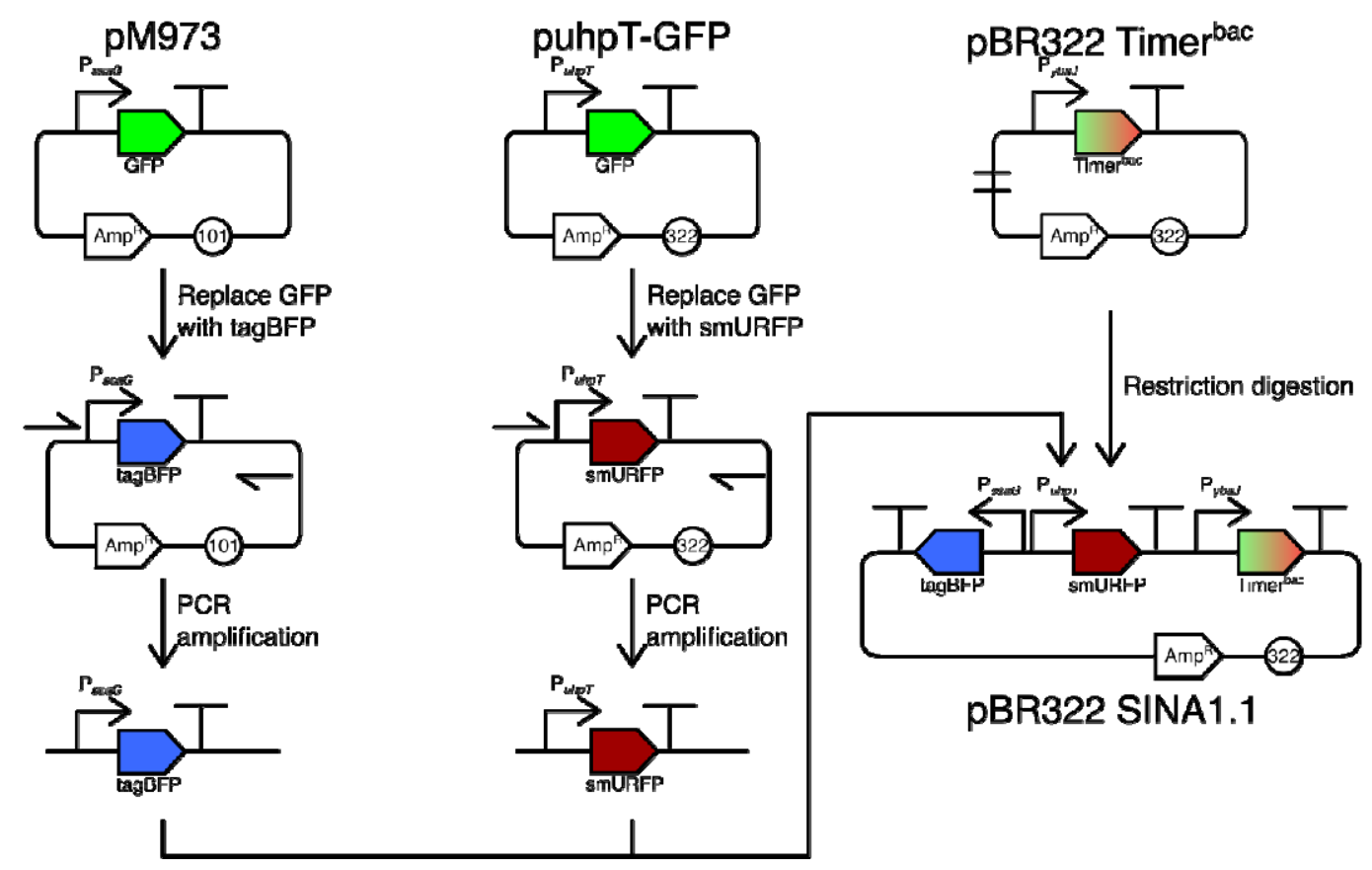

\section{Supplementary figure 1. Construction strategy of SINA1.1.}

The vacuolar and cytosolic modules were first individually tested with GFP (pM973 and puhpT-GFP), and then switched to tagBFP and smURFP, respectively. The vacuolar $\left(\mathrm{P}_{s s a G}\right.$-tagBFP) and cytosolic $\left(\mathrm{P}_{u h p T}\right.$-smURFP) modules were subsequently amplified and introduced into pBR322 Timer ${ }^{\text {bac }}$ between SphI and SalI sites to yield SINA1.1. 

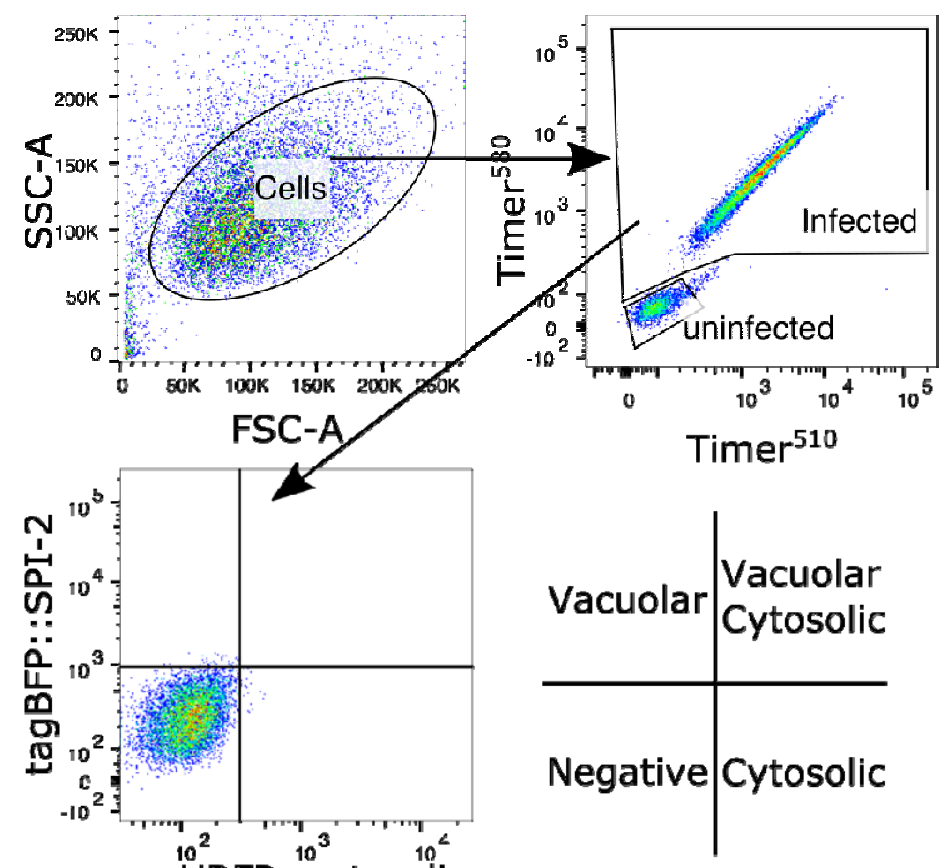

smURFP::cytosolic

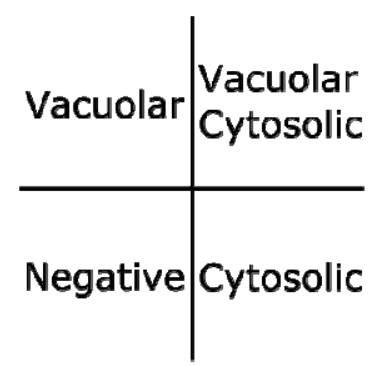

Supplementary figure 2. Gating strategy of SINA1.1 reporter system.

Analyzed events were first gated for "Cells" on SSC-A vs FSC-A plot to remove cell debris. In the "Cells" events, "Uninfected" population was gated by double-negative; "Infected" was gated by double-positive on $\operatorname{Timer}^{580}$ vs $\operatorname{Timer}^{510}$ plot. To gate for the basal intensity of SINA1.1 at $1 \mathrm{~h}$ pi, four quadrants were drawn in the "Infected" events on tagBFP::SPI-2 vs smURFP::cytosolic plot, where the biological interpretations of the four quadrants were denoted in the bottom-right sketch. 


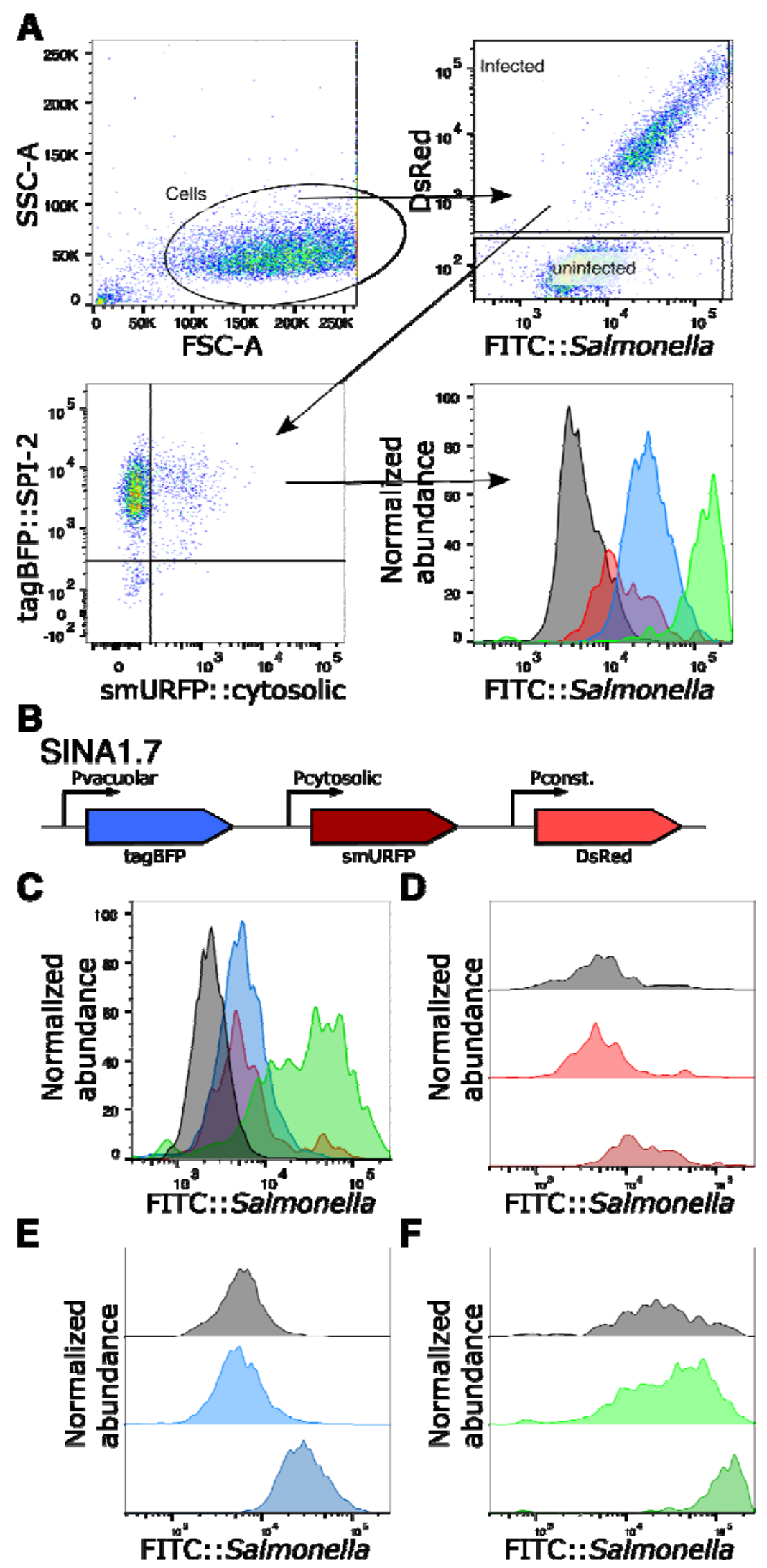




\section{Supplementary figure 3. Localization modules indicate subcellular localization of}

\section{$S$. Typhimurium.}

(A) Gating strategy for applying SINA1.7 for digitonin assay. HeLa cells were infected with SINA1.7-harboring wild type $S$. Typhimurium, and harvested at $6 \mathrm{~h}$ pi for analysis by flow cytometry. The events were first gated for "Cells" to remove cell debris and subsequently gated for "uninfected" and "infected" based on DsRed signal. The "infected" events were subsequently gated for $\mathrm{Vac}^{-} \mathrm{Cyt}$, $\mathrm{Vac}^{+} \mathrm{Cyt}^{-}$and $\mathrm{Vac}^{+} \mathrm{Cyt}^{+}$ on tagBFP::SPI-2 vs smURFP::cytosolic plot. The fluorescence profiles FITC::S. Typhimurium (after immunostaining using anti-S. Typhimurium antibody) of $\mathrm{Vac}^{-}$ $\mathrm{Cyt}^{-}, \mathrm{Vac}^{+} \mathrm{Cyt}^{-}$and $\mathrm{Vac}^{+} \mathrm{Cyt}^{+}$and "uninfected" were plotted as overlay histograms. The gating strategy displays a positive control sample treated with saponin. (B) Schematic diagram for the constructions of the SINA derivative SINA1.7, where Timer $^{\text {bac }}$ was replaced with DsRed as compared to SINA1.1. (C) Digitonin assay on SINA-1.7 harboring wild type $S$. Typhimurium-infected HeLa cells at $6 \mathrm{~h}$ pi, signal intensities of uninfected (black), $\mathrm{Vac}^{-} \mathrm{Cyt}^{-}$(red), $\mathrm{Vac}^{+} \mathrm{Cyt}^{-}$(blue) and $\mathrm{Vac}^{+} \mathrm{Cyt}^{+}$(green) populations immunostained against anti-S. Typhimurium. (D) Digitonin assay on SINA-1.7 harboring wild type $S$. Typhimurium infected HeLa cells at $6 \mathrm{~h}$ pi, signal intensity of $\mathrm{Vac}^{-} \mathrm{Cyt}^{-}$population unpermeabilized (black, negative control), permeabilized with digitonin (red) and saponin (maroon, positive control). (E) Digitonin assay on SINA-1.7 harboring wild type $S$. Typhimurium infected HeLa cells at $6 \mathrm{~h} \mathrm{pi}$, signal intensity of $\mathrm{Vac}^{+} \mathrm{Cyt}^{-}$population unpermeabilized (black, negative control), permeabilized with digitonin (blue) and saponin (navy, positive control). (F) Digitonin assay on SINA-1.7 harboring wild type $S$. Typhimurium infected HeLa cells at $6 \mathrm{~h}$ pi, signal intensity of $\mathrm{Vac}^{+} \mathrm{Cyt}^{+}$population unpermeabilized (black, negative control), permeabilized with digitonin (green) and saponin (dark Green, positive control). 


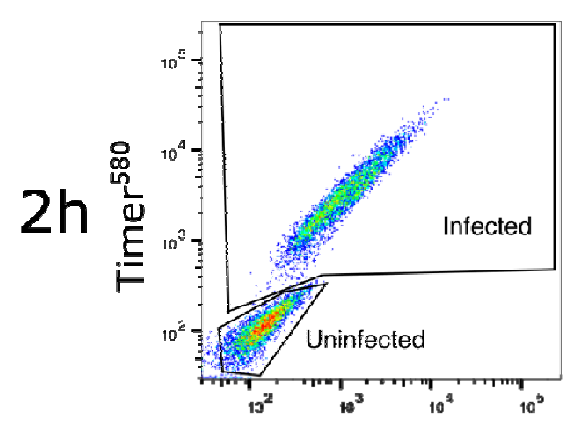

Timer $^{510}$

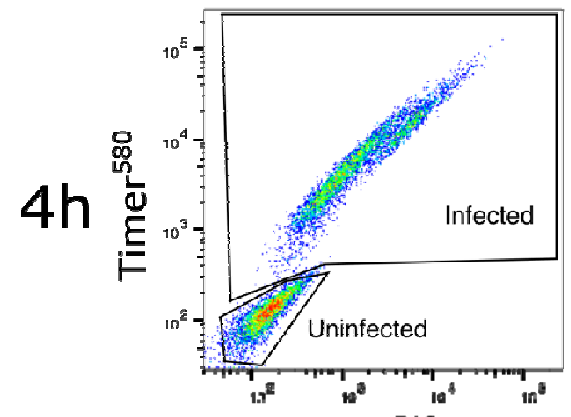

Timer $^{510}$

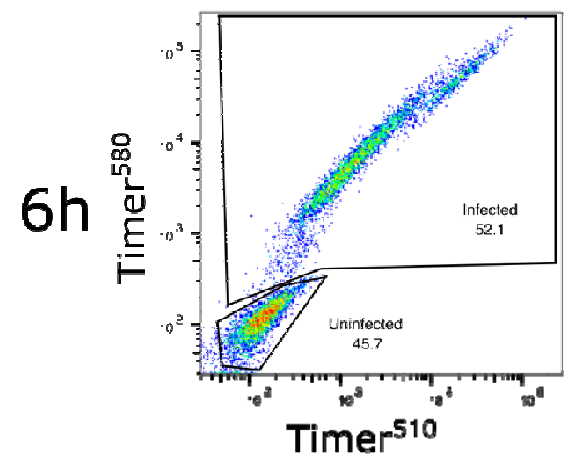

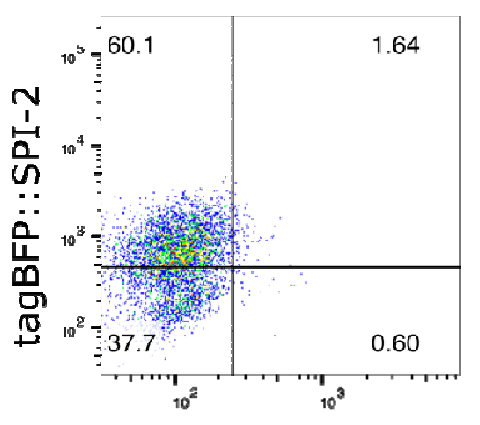

smURFP::cytosolic

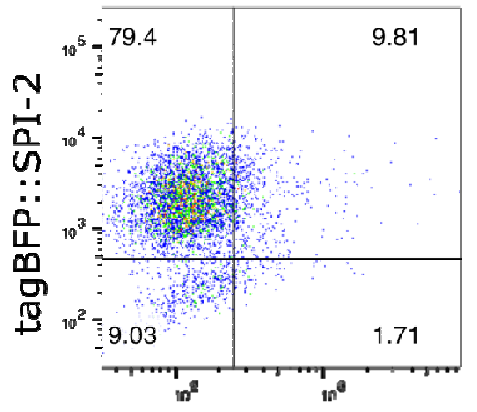

smURFP: : cytosolic

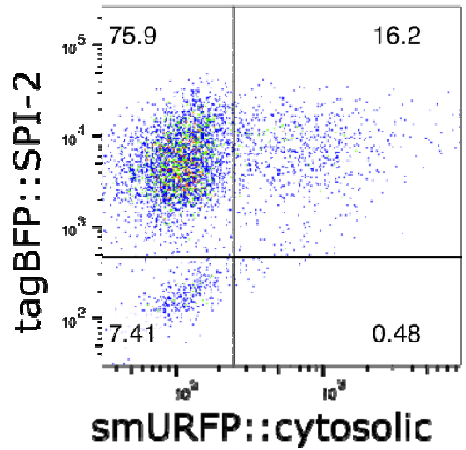

Supplementary figure 4. SINA1.1 performance in HeLa cells at $2 \mathrm{~h}, 4 \mathrm{~h}$ and $6 \mathrm{~h}$ pi.

HeLa cells were infected with wild type $S$. Typhimurium harboring SINA1.1, Infected cells were harvested and analyzed at time intervals of $2 \mathrm{~h}, 4 \mathrm{~h}$ and $6 \mathrm{~h}$ pi. (Left) Timer $^{\text {bac }}$ profile of total cells at $2 \mathrm{~h}$ (top), $4 \mathrm{~h}$ (middle) and $6 \mathrm{~h}$ (bottom) pi in HeLa cells. (Right) Fluorescence output of the localization module of infected cells at $2 \mathrm{~h}$ (top), $4 \mathrm{~h}$ (middle) and $6 \mathrm{~h}$ (bottom) pi in HeLa cells. 

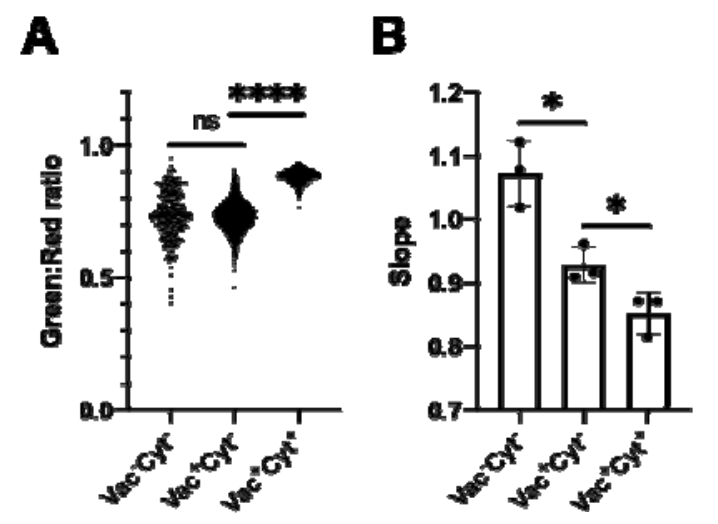

Supplementary figure 5. S. Typhimurium exhibits distinct replication rates and metabolism in HeLa cells.

HeLa cells were infected with SINA1.1-harboring $S$. Typhimurium and harvested at 6 $\mathrm{h}$ pi for analysis by flow cytometry. The three infected cell populations, $\mathrm{Vac}^{-} \mathrm{Cyt}^{-}$, $\mathrm{Vac}^{+} \mathrm{Cyt}{ }^{-}$and $\mathrm{Vac}^{+} \mathrm{Cyt}^{+}$on tagBFP::SPI-2 vs smURFP::cytosolic plot were backgated on Timer ${ }^{580}$ vs Timer ${ }^{510}$ plot. Timer ${ }^{580}$ and Timer $^{510}$ intensities were extracted from each event. (A) Quantification of Green:red ratio of $\mathrm{Vac}^{-} \mathrm{Cyt}$, $\mathrm{Vac}^{+} \mathrm{Cyt}^{-}$and $\mathrm{Vac}^{+} \mathrm{Cyt}^{+}$ population in Timer $^{\text {bac }}$ plot at $6 \mathrm{~h}$ pi. Green:red ratios were calculated by dividing Timer $^{510}$ by Timer $^{580}$ values, and plotted against infected cell populations. (B) Quantification of the slope of the best-fitted line of $\mathrm{Vac}^{-} \mathrm{Cyt}{ }^{-}, \mathrm{Vac}^{+} \mathrm{Cyt}^{-}$and $\mathrm{Vac}^{+} \mathrm{Cyt}^{+}$ population in Timer ${ }^{\text {bac }}$ plot at $6 \mathrm{~h}$ pi. For each population, a best-fitted line was plotted on the $\operatorname{Timer}^{580}$ vs $\operatorname{Timer}^{510}$ plot to extract the slopes for each infected cell populations. Unpaired t-tests were carried out for triplicated experiments, $* P<0.05$, $* * * * P<0.0001$, ns: not significant. 


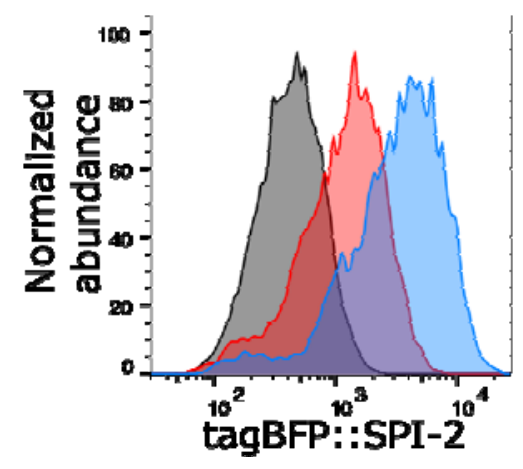

Supplementary figure 6. Dormant $S$. Typhimurium are observed as early as $2 \mathrm{~h}$ pi in HeLa cells.

HeLa cells were infected with SINA1.1-harboring $S$. Typhimurium, and harvested at $1 \mathrm{~h}, 2 \mathrm{~h}$ and $3 \mathrm{~h}$ pi for analysis by flow cytometry. The infected cells were gated and the fluorescence profiles of vacuolar submodule $\mathrm{P}_{s s a G}$-tagBFP at $1 \mathrm{~h}$ (black), $2 \mathrm{~h}$ (red) and $3 \mathrm{~h}$ (blue) pi were plotted as overlaying histograms. 


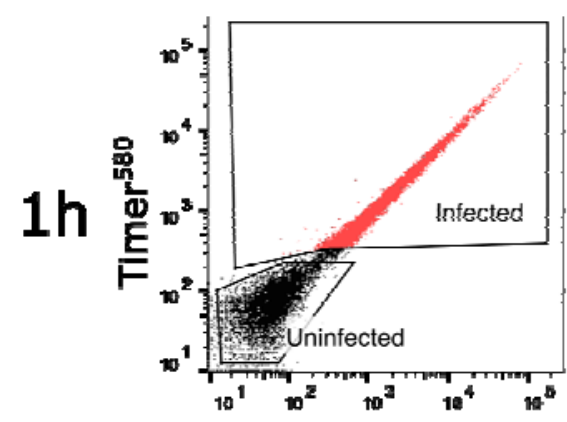

Timer ${ }^{510}$
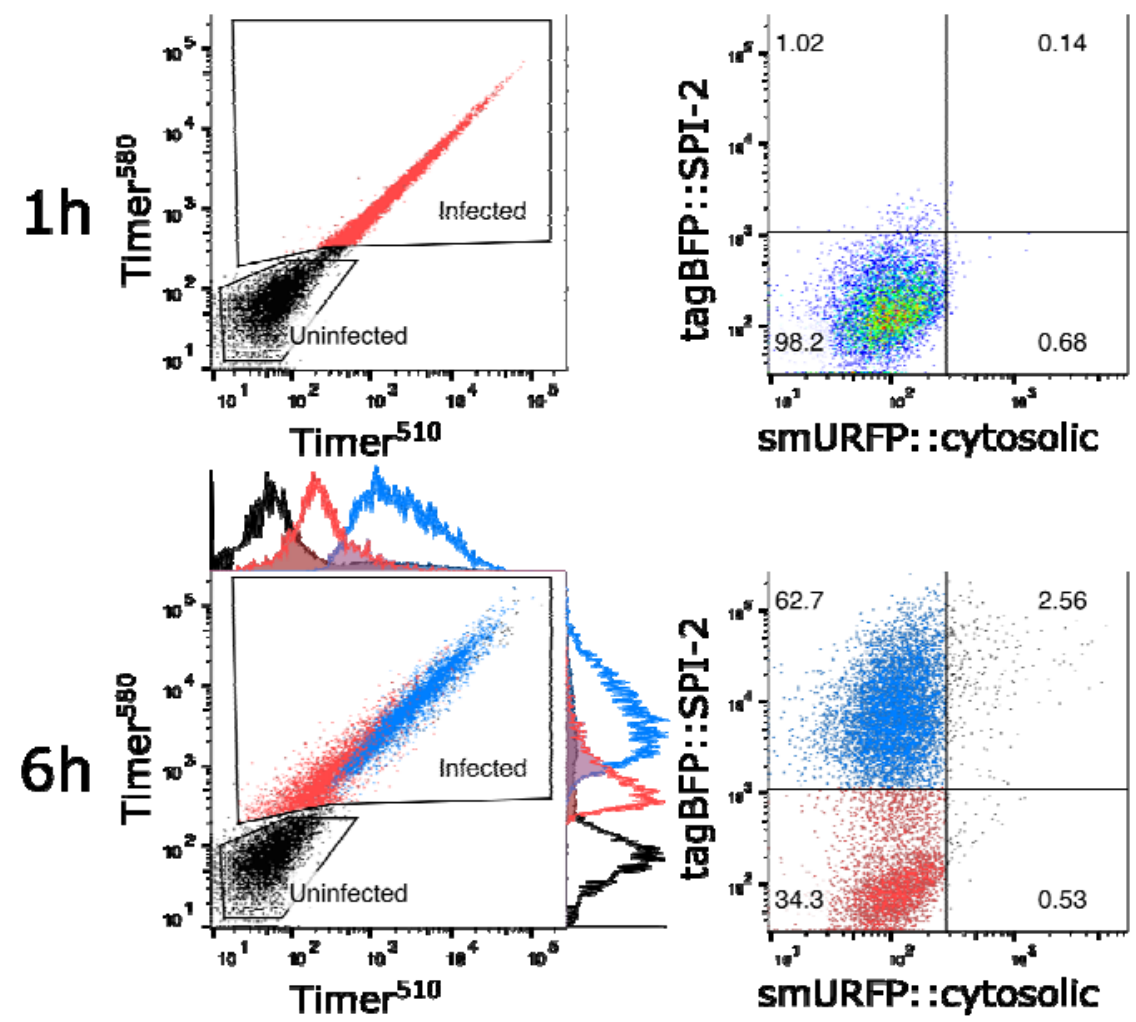

Supplementary figure 7. Performance of SINA1.1 in Caco-2 cells.

Polarized Caco-2 monolayers were infected with SINA1.1-harboring $S$. Typhimurium and harvested at $1 \mathrm{~h}$ and $6 \mathrm{~h}$ pi for analysis by flow cytometry. (Left) Timer ${ }^{\text {bac }}$ profile of $\mathrm{Vac}^{-} \mathrm{Cyt}^{-}$(red) and $\mathrm{Vac}^{+} \mathrm{Cyt}^{-}$(blue) populations and total cells (black) at $1 \mathrm{~h}$ (top) and $6 \mathrm{~h}$ (bottom) pi in Caco-2 cells. (Right) Distribution of $\mathrm{Vac}^{-} \mathrm{Cyt}^{-}$and $\mathrm{Vac}^{+} \mathrm{Cyt}^{-}$ populations at $1 \mathrm{~h}$ (top) and $6 \mathrm{~h}$ (bottom) pi in polarized Caco-2 cells. 

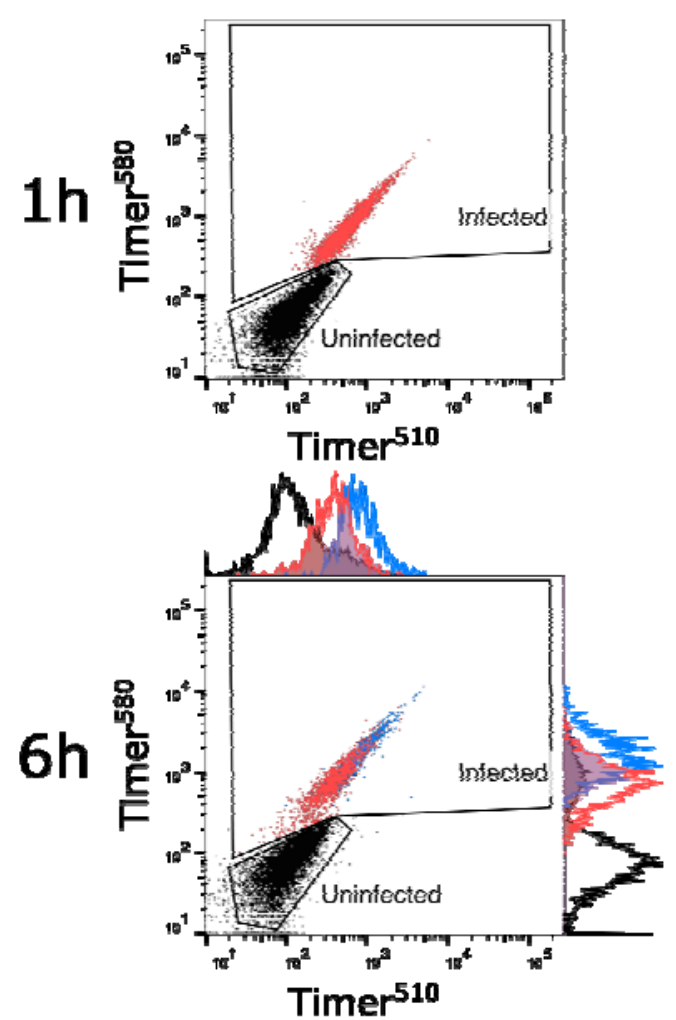
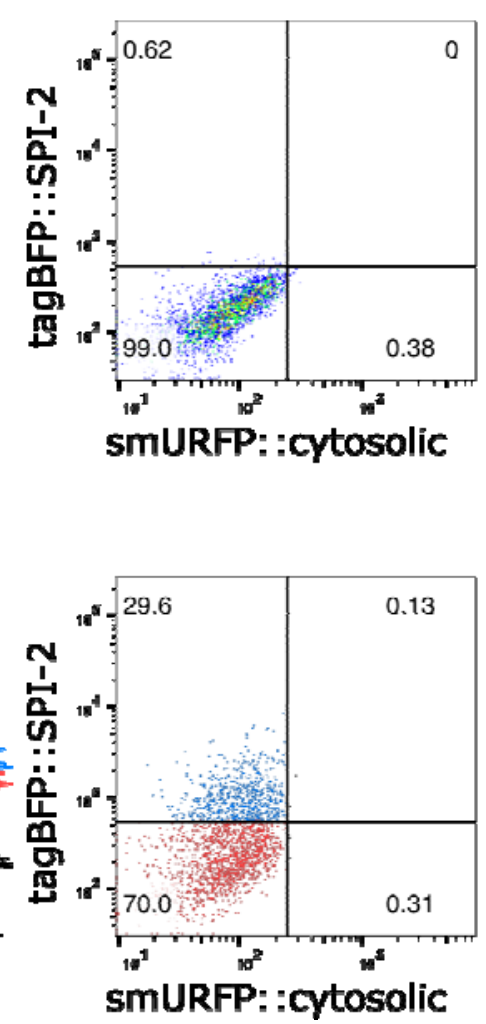

Supplementary figure 8. Performance of SINA1.1 in THP-1 cells.

Differentiated THP-1 cells were infected with SINA1.1-harboring S. Typhimurium and harvested at $1 \mathrm{~h}$ and $6 \mathrm{~h}$ pi for analysis by flow cytometry. (Left) Timer ${ }^{\text {bac }}$ profile of $\mathrm{Vac}^{-} \mathrm{Cyt}^{-}$(red) and $\mathrm{Vac}^{+} \mathrm{Cyt}^{-}$(blue) populations and total cells (black) at $1 \mathrm{~h}$ (top) and $6 \mathrm{~h}$ (bottom) pi in THP-1 cells. (Right) Distribution of $\mathrm{Vac}^{-} \mathrm{Cyt}^{-}$and $\mathrm{Vac}^{+} \mathrm{Cyt}^{-}$ populations at $1 \mathrm{~h}$ (top) and $6 \mathrm{~h}$ (bottom) pi in differentiated THP-1 cells. 


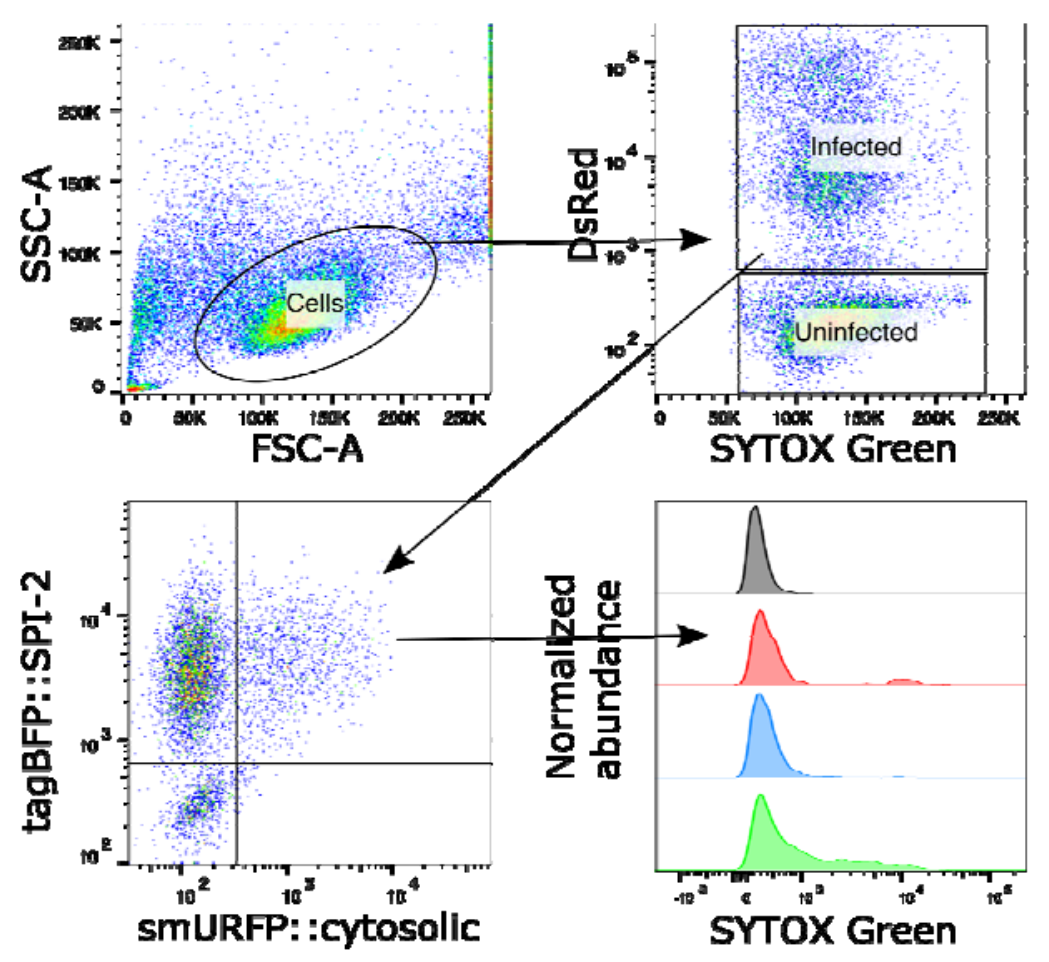

Supplementary figure 9. Infected cells harboring dormant $S$. Typhimurium are viable.

HeLa cells were infected with SINA1.7 harboring S. Typhimurium, harvested at $6 \mathrm{~h}$ pi and stained with SYTOX Green and analyzed by flow cytometry. The infected cells were gated and the fluorescence profiles of SYTOX Green in uninfected cell (black), $\mathrm{Vac}^{-} \mathrm{Cyt}^{-}$(red), $\mathrm{Vac}^{+} \mathrm{Cyt}^{-}$(blue) and $\mathrm{Vac}^{+} \mathrm{Cyt}^{+}$(green) were plotted as offset histograms. 


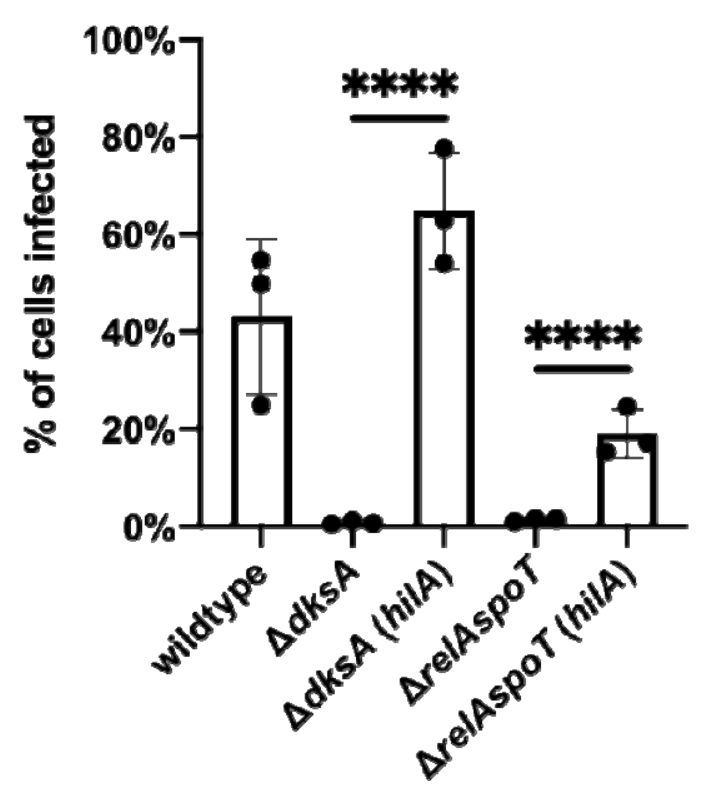

Supplementary figure 10. Ectopic expression of hilA rescues the loss of invasiveness.

HeLa cells were infected with various $S$. Typhimurium strains and harvested at $6 \mathrm{~h}$ pi for flow cytometry analysis. The losses of invasiveness in $\triangle d k s A$ and $\triangle$ relAspoT mutants are rescued by ectopic expression of hilA from the arabinose inducible cassette in SINA1.9. Unpaired t-test was carried out for triplicated experiments, $* * * * P<0.0001$. 
bioRxiv preprint doi: https://doi.org/10.1101/2020.11.14.382689; this version posted November 15,2020 . The copyright holder for this preprint (which was not certified by peer review) is the author/funder, who has granted bioRxiv a license to display the preprint in perpetuity. It is made available under aCC-BY-NC-ND 4.0 International license.

\section{Supplementary movies}

The manuscript contains 3 supplementary movies. The figures to which they refer are indicated in the titles of the movies. 